\title{
Development and validation of a dynamic range-extended LC-MS/MS multi-analyte method for 11 different postmortem matrices for redistribution studies applying solvent calibration and additional ${ }^{13} \mathrm{C}$ isotope monitoring
}

\author{
Sandra N. Staeheli ${ }^{1} \cdot$ Michael Poetzsch $^{1}$ - Thomas Kraemer $^{1}$ • Andrea E. Steuer ${ }^{1}$
}

Received: 16 July 2015 / Revised: 29 August 2015 / Accepted: 1 September 2015 / Published online: 22 September 2015

(C) Springer-Verlag Berlin Heidelberg 2015

\begin{abstract}
Postmortem redistribution (PMR) is one of numerous problems in postmortem toxicology making correct interpretation of measured drug concentrations difficult or even impossible. Time-dependent PMR in peripheral blood and especially in tissue samples is still under-explored. For further investigation, an easy applicable method for the simultaneous quantitation of over 80 forensically relevant compounds in 11 different postmortem matrices should be developed and validated overcoming the challenges of high inter-matrix and intra-matrix concentration variances. Biopsy samples $(20 \mathrm{mg})$ or body fluids $(20 \mu \mathrm{L})$ were spiked with an analyte mix and deuterated internal standards, extracted by liquidliquid extraction, and analyzed by liquid chromatography tandem mass spectrometry (LC-MS/MS). For highest applicability, an easy solvent calibration was used. Furthermore, timeconsuming dilution of high concentration samples showing detector saturation was circumvented by two overlapping calibration curves using ${ }^{12} \mathrm{C}$ isotope monitoring for low concentrations and ${ }^{13} \mathrm{C}$ isotopes for high concentration, respectively. The method was validated according to international guidelines with modifications. Matrix effects and extraction efficiency were strongly matrix and analyte dependent. In general, brain and adipose tissue produced the highest matrix effects, whereas cerebrospinal fluid showed the least matrix
\end{abstract}

Electronic supplementary material The online version of this article (doi:10.1007/s00216-015-9023-5) contains supplementary material, which is available to authorized users.

Andrea E. Steuer

andrea.steuer@irm.uzh.ch

1 Department of Forensic Pharmacology and Toxicology, Zurich Institute of Forensic Medicine, University of Zurich, Winterthurerstrasse 190/52, 8057 Zurich, Switzerland effects. Accuracy and precision results were rather matrix independent with some exceptions. Despite using an external solvent calibration, the accuracy requirements were fulfilled for 66 to $81 \%$ of the 83 analytes. Depending on the matrix, $75-93 \%$ of the analytes showed intra-day precisions at $<20 \%$. ${ }^{12} \mathrm{C}$ and ${ }^{13} \mathrm{C}$ calibrations gave comparable results and proved to be a useful tool in expanding the dynamic range.

Keywords LC-MS/MS · Postmortem - Alternative matrices · ${ }^{13} \mathrm{C}$ isotope calibration $\cdot$ Expanded dynamic range

\section{Introduction}

Postmortem redistribution (PMR) is a well-recognized phenomenon in forensic toxicology, which can complicate interpretation of drug concentrations and conclusions on the cause of death in medico-legal investigations. The PMR phenomenon combines concentration changes after death due to degradation, neo-formation, or diffusion processes (e.g., from high drug concentrations in the gastrointestinal tract or the lungs) and seems to be strongly dependent on analyte characteristics and the individual case circumstances such as the distribution of the analyte at time of death [1]. Furthermore, the time between death and sampling time point should be of major importance. Blood from peripheral sites - usually obtained from the femoral vein - is mostly agreed to be the matrix of choice for quantitation of xenobiotics because of its distance from central organs and the gastrointestinal tract [2]. However, even peripheral blood was shown to exhibit massive time-dependent PMR with drug concentrations decreasing or increasing up to $300 \%$ [3]. Extensive blood clotting, collapse of the veins, putrefaction processes, or exsanguination can lead to unavailability of blood for analysis. If 
no blood at all is available, alternative matrices such as liver, kidney, muscle, lung, etc., have to be considered for toxicological analysis [4]. However, the interpretation of quantitation results in alternative postmortem matrices provides even more pitfalls due to lacking reference values or unpredictable PMR processes. PMR within solid tissues can only be assumed based on site-dependent concentration differences in samples taken at autopsy. Nevertheless, information about time-dependent redistribution in solid tissues is needed as these matrices are used for forensic investigations. Collection of solid tissue samples at more than one time point is only possible in the time window between admission of the body at the mortuary and autopsy with biopsy sampling before autopsy representing the only practicable strategy. Quantitation in biopsies is not a common practice in forensic toxicology. Only a few papers focused on quantitation in biopsy samples, e.g., in renal biopsies from rats and humans for the immunosuppressant drugs mycophenolic acid and tacrolimus. Tissue samples were homogenized either mechanically or enzymatically and then underwent liquid-liquid extraction (LLE). Sensitive instrumentation such as liquid chromatography (LC) coupled to mass spectrometry (MS) is required as only minimal sample amount of about $20 \mathrm{mg}$ /biopsy is usually available $[5,6]$.

In postmortem toxicology, substances of different pharmacological classes such as opiates, benzodiazepines, antidepressants, neuroleptics, or drugs of abuse are of interest with often limited knowledge on consumed drugs prior to analysis. As ideal sample preparation and analytical instrumentation may vary between matrices and substance classes, often different analytical methods are used for quantitation [7]. However, sufficient sample amount is necessary which is often difficult for postmortem samples especially with biopsy samples. Multi-analyte procedures aiming at covering a multitude of relevant compounds seems to represent an interesting alternative. Unspecific extraction methods such as protein precipitation (PP) allow simultaneous extraction of various substance classes and are fast and cheap. However, extract purity after $\mathrm{PP}$ is low especially with postmortem matrices and tissue extractions which in turn can lead to severe matrix effects [8]. These problems are reduced using more sophisticated LLE or solid-phase extractions (SPE) [9]. On the other hand, LLE and SPE are more specific and may not work for simultaneous extraction of various substance classes. Several methods covering a large amount of analytes have been published in recent years and are summarized in [10].

Another major challenge in quantifying that many compounds with different therapeutic and/or toxic concentration ranges in different matrices in only one analytical run is to find calibration models which can handle the huge dynamic range without source or detector saturation of the MS. Different strategies to overcome these problems have been applied including the use of offset voltage parameters, less-sensitive product ions, polarity switching, or ${ }^{13} \mathrm{C}$ product ions [11, 12]. The naturally occurring ${ }^{13} \mathrm{C}$ isotope is usually about $1.1 \%$ of all carbon atoms producing a much lower MS signal compared with ${ }^{12} \mathrm{C}$. Using the ${ }^{13} \mathrm{C}$ isotope as precursor ion can help avoid saturation effects. The ${ }^{13} \mathrm{C}$ isotope is stable, and its distribution underlies geological differences [13]. To the best of our knowledge, only Bergeron et al. tested the use of a ${ }^{13} \mathrm{C}$ isotope as precursor for the simultaneous quantitation of mebeverine and its metabolite as both show large differences in their therapeutic plasma concentration ranges. However, in their case, application of ${ }^{13} \mathrm{C}$ precursors did not give the desired result and the strategy of polarity switching was used instead for final quantitation [11].

Although forensic toxicologists are aware of the PMR effect, it is still under-explored. Especially systematic studies on time-dependent postmortem changes are limited and are difficult to conduct due to practical and even more important due to ethical reasons. To provide better insight into timedependent PMR processes in blood as well as in different postmortem tissues, a novel, sensitive method should be developed and validated using LC-MS/MS allowing simultaneous quantitation in low amount of biopsy samples of different postmortem tissues for a multitude of over 80 forensically relevant compounds. This method should create the prerequisites for further studies on time-dependent PMR.

\section{Materials and methods}

\section{Chemicals and reagents}

Methanolic or acetonitrilic solutions $(1 \mathrm{mg} / \mathrm{mL})$ of 6 monoacetylmorphine (MAM), agomelatine, alprazolam, amisulpride, amitriptyline, amphetamine, nortriptyline, aripiprazole, benzoylecgonine (BEC), bromazepam, bupropion, hydroxybupropion (OH-bupropion), chlorprothixene, clonazepam, 7-aminoclonazepam, citalopram, clomipramine, norclomipramine, clozapine, norclozapine, cocaine, codeine, diazepam, dihydrocodeine (DHC), doxepine, nordoxepine, duloxetine, ecgonine methyl ester (EME), fentanyl, fluoxetine, norfluoxetine, flupentixol, flunitrazepam, 7-aminoflunitrazepam, flurazepam, $\mathrm{N}$ desalkylflurazepam, hydroxyethylflurazepam (OHflurazepam), haloperidol, hydrocodone (HC), hydromorphone (HM), imipramine, ketamine, levomepromazine, lorazepam, meta-chlorophenylpiperazine (mCPP), methadone, 1,5-dimethyl-3,3-diphenylpyrrolidine (EDDP), methamphetamine, 3,4-methylenedioxyamphetamine (MDA), 3,4methylenedioxy- $N$-ethylamphetamine (MDEA), 3,4methylendioxymethamphetamine (MDMA), methylphenidate (MPH), midazolam, $\alpha$-hydroxymidazolam (OH-midazolam), mirtazapine, morphine, nitrazepam, nordazepam, olanzapine, opipramol, oxazepam, oxycodone, oxymorphone, 
paroxetine, perazine, pethidine, pipamperone, promazine, quetiapine, risperidone, 9-hydroxyrisperidone (OH-risperidone), sertraline, trazodone, temazepam, tramadol, $O$ desmethyltramadol (ODMT), triazolam, trimipramine, venlafaxine, $O$-desmethylvenlafaxine (ODMV), zolpidem and zopiclone and methanolic solutions of the deuterated internal standards (IS; $0.1 \mathrm{mg} / \mathrm{mL}$ ), MAM-d3, alprazolam-d5, amisulpride-d5, amitriptyline-d3, amphetamine-d6, aripiprazole-d8, citalopram-d6, clomipramine-d3, clonazepam-d4, 7-aminoclonazepam-d4, clozapine-d3, cocaine-d3, BEC-d3, EME-d3, codeine-d3, diazepam-d5, duloxetine-d3, fentanyl-d5, flunitrazepam-d3, 7-aminoflunitrazepam-d3, fluoxetine-d6, norfluoxetine-d6, $N$-desalkylflurazepam-d4, flurazepam-OH-d4, haloperidol-d4, ketamine-d4, lorazepamd4, mCPP-d8, MDMA-d5, methadone-d9, methamphetamine- $\mathrm{d} 9$, methylphenidate- $\mathrm{d} 9$, midazolam- $\mathrm{d} 4$, midazolam-OH-d4, morphine-d3, nordazepam-d5, oxazepamd5, paroxetine-d6, pethidine-d4, pipamperone-d10, quetiapine-d8, risperidone-d4, temazepam-d5, tramadold3, trazodone-d6, triazolam-d4, trimipramine-d3, venlafaxine-d6, zolpidem-d6 and zopiclone-d4 were obtained from AdipoGen (Liestal, Switzerland), Lipomed (Arlesheim, Switzerland), LGC (Wesel, Germany), or Cerilliant (delivered by Sigma-Aldrich, Buchs, Switzerland). Water was purified with a Purelab Ultra Millipore filtration unit (Labtech, Villmergen, Switzerland) and acetonitrile of HPLC grade was obtained from Fluka (Buchs, Switzerland). All other chemicals used were from Merck (Zug, Switzerland) and of the highest grade available.

\section{Biosamples}

Human blank whole blood samples from the femoral veins (femoral blood (FB)) and the heart (heart blood (HB)), cerebrospinal fluid (CSF) from the lumbar spine, urine, muscle, liver, kidney, lung, spleen, brain tissue, and adipose tissue (AT) were used for method development and validation. They were obtained from postmortem cases that were submitted to the authors' laboratory and autopsied by order of the state attorney. Ethical approval was obtained by the Cantonal Ethics Committee of Zurich (approval number 42.2005).

\section{Sample preparation}

Body fluid and tissue samples were extracted by a two-step LLE; $20 \mathrm{mg}$ body tissue (muscle, liver, kidney, lung, spleen, adipose, or brain tissue) were weighed into $2 \mathrm{~mL}$ plastic tubes containing metal beads and homogenized using a Fast Prep ${ }^{\circledR}$ 24 Instrument (MP Biomedicals, Illkirch, France). Twenty microliters of body fluid (blood, CSF, or urine) were extracted in a 2-mL Eppendorf tube (Schoenenbuch, Switzerland). To each sample (homogenized tissues or body fluids), $50 \mu \mathrm{L}$ IS mixture (concentrations are given in the Electronic supplementary material (ESM) Table S1) and $10 \mu \mathrm{L}$ spiking solution were added. The first LLE was performed by adding $250 \mu \mathrm{L}$ of $0.2 \mathrm{M}$ phosphate buffer ( $\mathrm{pH} 7.4$ ) and $0.8 \mathrm{~mL}$ of butyl acetate/ethyl acetate (BuAc/EtAc; 1:1, v/v). The mixture was shaken for $10 \mathrm{~min}$ and centrifuged (5 min, 10,000 rpm), and $750 \mu \mathrm{L}$ supernatant were transferred into an autosampler vial. To the remaining sample, $100 \mu \mathrm{L}$ of $0.1 \mathrm{M} \mathrm{NaOH}$ was added (pH 13.5) and the second LLE was performed by adding another $0.8 \mathrm{~mL}$ of BuAc/EtAc $(1: 1, v / v)$, shaking, and centrifugation as described above. Eight hundred microliters of supernatant was added to the first extract, and after addition of $25 \mu \mathrm{L}$ formic acid $(20 \%, v / v)$, evaporated to dryness under a gentle stream of nitrogen at $40{ }^{\circ} \mathrm{C}$. The sample was reconstituted in $60 \mu \mathrm{L}$ mobile phase (eluent $\mathrm{A} / \mathrm{B}$ 90:10 $(v / v)$, see below) and analyzed by LC-MS/MS.

\section{LC-MS/MS settings}

The analysis was performed using a Thermo Fisher Ultimate 3000 UHPLC system (Thermo Fisher, San Jose, California, USA) coupled to an Sciex 5500 QTrap linear ion-trap quadrupole mass spectrometer (Sciex, Darmstadt/Germany). The LC settings were as follows: Phenomenex (Aschaffenburg, Germany) Synergy Polar RP column $(100 \times 2.0 \mathrm{~mm}$, $2.5 \mu \mathrm{m}$ ), gradient elution with $10 \mathrm{mM}$ ammonium formate buffer in water containing $0.1 \%(v / v)$ formic acid $(\mathrm{pH} 3.5$, eluent A), and acetonitrile containing $0.1 \%(v / v)$ formic acid (eluent B). Start conditions were $10 \%$ B for 1 min with a flow rate of $0.5 \mathrm{~mL} / \mathrm{min}, 1-10 \mathrm{~min}$ to $25 \% \mathrm{~B}$ and $0.6 \mathrm{~mL} / \mathrm{min}, 10$ $17 \mathrm{~min}$ concave upward to $95 \% \mathrm{~B}$, hold at $95 \% \mathrm{~B}$ for $0.5 \mathrm{~min}$, at $17.5-18.5 \mathrm{~min}$ re-equilibrating to $10 \% \mathrm{~B}$ and $0.5 \mathrm{~mL} / \mathrm{min}$. Injection volume was $10 \mu \mathrm{L}$.

The Turbo $\mathrm{V}$ ion source equipped with a stainless steel electrode $(100 \mu \mathrm{m}$ internal diameter) was operated in positive electrospray ionization (ESI) mode with the following MS conditions: gas 1, nitrogen (50 psi); gas 2 , nitrogen (60 psi); ion spray voltage, $5500 \mathrm{~V}$; ion-source temperature, $450{ }^{\circ} \mathrm{C}$; curtain gas, nitrogen ( $30 \mathrm{psi}$ ); and collision gas, medium. The MS was operated in the advanced scheduled multiple reaction monitoring (MRM) mode using three transitions for each analyte. The third transition corresponded to the ${ }^{13} \mathrm{C}$ isotope of the first transition. The mass spectrometer settings for MRM 3 were identical to those of MRM 1, except the Q1 and Q3 masses were set $1 \mathrm{Da}$ higher. For each analyte transition, an intensity threshold was defined. For the ISs, 1 MRM transition was used. The MS settings for each analyte are given in ESM Table S2. The MS was controlled by Analyst ${ }^{\circledR}$ 1.6.2 software (Sciex, Darmstadt/Germany). Quantitation was performed with MultiQuant ${ }^{\circledR}$ 2.1.1 software (Sciex, Darmstadt/ Germany). 


\section{Method validation}

\section{Preparation of calibration samples}

Methanolic or acetonitrilic solutions $(1 \mathrm{mg} / \mathrm{mL})$ for each analyte were used as stock solutions for calibration spiking solutions. Working solutions $(0.1$ and $0.01 \mathrm{mg} / \mathrm{mL})$ were prepared by dilution from each stock solution in methanol or acetonitrile, depending on the solvent delivered in. For antidepressants and neuroleptics (ADN), two spiking solutions in methanol (d0-mix 1 ADN MeOH and d0-mix 2 ADN MeOH) and two spiking solutions in acetonitrile (d0-mix $1 \mathrm{ADN} A C N$ and d0-mix 2 ADN ACN) for calibration standards were prepared by mixing appropriate amounts of the corresponding stock or working solutions as described previously [14]. For all other analytes, two spiking solutions in methanol (d0-mix 1 rest $\mathrm{MeOH}$ and d0-mix 2 rest $\mathrm{MeOH}$ ) were prepared.

Different amounts of the d0-mixes 1 or 2 were diluted to a total volume of $5 \mathrm{~mL}$ methanol or acetonitrile to give the final calibration spiking solutions (Cal 1-13 ADN in methanol and Cal 1-13 ADN in acetonitrile and Cal 1-13 rest in methanol) in a final concentration two times higher than the aimed target blood or tissue concentration.

The final calibration samples were prepared without matrix; $10 \mu \mathrm{L}$ of each calibration spiking solution was mixed with IS and $25 \mu \mathrm{L}$ formic acid $(20 \%, v / v)$ in an autosampler vial, dried at $40{ }^{\circ} \mathrm{C}$ under nitrogen and reconstituted in $60 \mu \mathrm{L}$ eluent $\mathrm{A}$ and $\mathrm{B}(90: 10, v / v)$. The final calibration ranges are given in ESM Table S3 for each analyte.

\section{Preparation of quality control samples}

Stock, working, and spiking solutions were prepared as described above from different stock solutions. Different amounts of either methanolic or acetonitrilic stock or working solutions were mixed to the quality control (QC) spiking solution (low, med, high, ${ }^{13} \mathrm{C}$ in methanol or acetonitrile), in a final concentration two times higher than the corresponding blood/tissue concentrations. The concentration of QC low was defined between calibrators 1 and 2, QC med between calibrators 5 and 6, QC high between calibrators 9 and 10, and QC ${ }^{13} \mathrm{C}$ between calibrators 12 and 13 . QC samples were prepared from $20 \mu \mathrm{L}$ analyte-free body fluid or $20 \mathrm{mg}$ analyte-free body tissue and $10 \mu \mathrm{L}$ of the corresponding fortifying solution in methanol and $10 \mu \mathrm{L}$ of the corresponding fortifying solution in acetonitrile. The final QC concentrations are given in ESM Table S3 for each analyte.

\section{Selectivity and crosstalk}

Five blank matrix samples from different sources were analyzed for peaks interfering with the detection of analytes or IS for $\mathrm{FB}, \mathrm{HB}, \mathrm{CSF}$, urine, muscle, liver, kidney, lung, spleen, brain, and AT. A methanolic solution of the IS mixture was analyzed to check for appropriate IS purity and presence of native analytes. A methanolic solution of each analyte was injected separately at concentrations of 1 or $10 \mu \mathrm{g} / \mathrm{mL}$ and checked for interfering peaks. Analytes which showed interfering peaks were then tested at their $\mathrm{QC}$ high and $\mathrm{QC}{ }^{13} \mathrm{C}$ concentration.

\section{Calibration model}

Calibrators 1-13 were divided into two partly overlapping calibrations, conventional ${ }^{12} \mathrm{C}$ calibration with calibrators $1-$ 10 and isotope $\left({ }^{13} \mathrm{C}\right)$ calibration with calibrators $8-13$. In the ${ }^{12} \mathrm{C}$ calibration, MRM 1 ( ${ }^{12} \mathrm{C}$ isotope) was used as quantifier; in the ${ }^{13} \mathrm{C}$ calibration, MRM $3\left({ }^{13} \mathrm{C}\right.$ isotope of MRM 1) was used as quantifier.

Calibration curves (single measurement per level at four validation days) were prepared with each batch of validation samples. The regression lines for both calibrations ${ }^{12} \mathrm{C}$ and ${ }^{13} \mathrm{C}$ were calculated using a simple linear model with $1 / x$ weighting. Requirements were back-calculated calibrator concentration of $\pm 30 \%$ of target and sufficient accuracy $( \pm 30 \%$ as described below for bias and precision) of the analyzed QC samples. Only for analytes that did not fulfill these criteria, additionally linear $1 / x^{2}$, quadratic or semi-logarithmic quadratic weighted $1 / x$, and $1 / x^{2}$ least-square regression model were tested. From those, a final calibration model was chosen, which yielded the lowest bias and precision data over the four validation days.

\section{Matrix effects and extraction efficiency}

FB, HB, CSF, urine, muscle, liver, kidney, lung, spleen, AT, and brain tissue from five different postmortem cases were used for the matrix-effect (ME) and extraction-efficiency (EE) experiments at QC med concentration. The experiments were performed according to Matuszewski et al. [15]. Briefly, three sets of samples (X, Y, Z) were prepared. Group $\mathrm{X}$ was spiked with IS and QC med spiking solution before extraction, while group $\mathrm{Y}$ was spiked after extraction. Group Z consisted of five QC med samples without matrix. EE and ME were calculated using Eqs. 1 and 2, respectively.

$$
\begin{aligned}
& \% \mathrm{EE}=\frac{X}{Y} * 100 \\
& \% \mathrm{ME}=\frac{Y}{Z} * 100
\end{aligned}
$$

Where $X=$ analyte peak area in group $X ; Y=$ analyte peak area in group $\mathrm{Y}$; and $Z=$ analyte peak area in group $\mathrm{Z}$. 


\section{Accuracy and precision}

QC samples (low, high, ${ }^{13} \mathrm{C}$ ) were analyzed according to the procedures described above in duplicates on each of 4 days. Bias was calculated as the percent deviation of the mean calculated concentration at each concentration level from the corresponding theoretical concentration. Intra-day $\left(\mathrm{RSD}_{\mathrm{R}}\right)$ and inter-day $\left(\mathrm{RSD}_{\mathrm{T}}\right)$ precisions were calculated as relative standard deviation (RSD) according to [16].

\section{Limits}

The lower limits of quantitation (LOQ) were defined as the concentrations of the first calibrator fulfilling the criteria of signal to noise 1:10 determined via peak heights. The limits of detection (LOD) were not systematically investigated.

\section{Comparison of accuracy between ${ }^{12} \mathrm{C}$ and ${ }^{13} \mathrm{C}$ calibration}

QC low was only calculated using ${ }^{12} \mathrm{C}$ calibration, QC ${ }^{13} \mathrm{C}$ was only calculated using ${ }^{13} \mathrm{C}$ calibration. QC high accuracy was calculated in both calibrations and was compared by Bland-Altman plots. For that, the average concentration of QC high calculated via ${ }^{12} \mathrm{C}$ and ${ }^{13} \mathrm{C}$ calibration over 4 days (see "Accuracy and precision") was plotted versus the difference percentage between ${ }^{12} \mathrm{C}$ and ${ }^{13} \mathrm{C}$ calibrations.

\section{Results and discussion}

Although forensic toxicologists are aware of the PMR effect, it is still under-explored. To provide better insight into timedependent PMR processes in blood and especially in different postmortem tissues, biopsy samples should be collected at admission of the corpse at the mortuary and again during autopsy and compared for a novel study. Reliable conclusion of results can only be drawn if the applied analytical methodologies can be shown to be precise enough to be able to address concentration changes as actual postmortem changes compared with analytical precision. Therefore, the aim of the present study was the development and validation of a rather simple and applicable LC-MS/MS method in 11 different postmortem specimens, with special focus on acceptable intra-day precision.

\section{Sample preparation}

Collection of solid tissue samples at more than one time point without opening of the corpse is only possible using biopsy sampling. Quantitation of drugs in postmortem biopsies has not been published in the field of forensic toxicology yet. Generally, only few publications exist about quantitation of small molecules in tissue biopsies. In these studies, not only was sample preparation started with mechanical homogenization in most of the cases [6, 17] but also enzymatic and chemical digestion was described [5, 18]. As digestion techniques were rather time consuming and may lead to stability problems for some analytes, mechanical homogenization was chosen for the present study. Due to small sample sizes of maximum $20 \mathrm{mg}$ body tissue collected during biopsy or $20 \mu \mathrm{L}$ body fluid, only one extract could be generated from one sample. Therefore, a rather unspecific sample preparation was required allowing simultaneous extraction of a large number of analytes. In forensic toxicology, sample preparation of postmortem matrices consists either of a PP, LLE, or SPE step in most of the cases [19-21]. LLE or SPE are more time consuming compared with the faster and rather unspecific PP but can reduce ME more efficiently [9, 22]. Therefore, a compromise had to be found allowing simultaneous extraction of a multitude of forensically relevant compounds with a high variety in physical-chemical properties but with still acceptable ME in postmortem matrices. In preliminary experiments, PP and different SPE and LLE procedures were compared in terms of EE and ME (data not shown). LLE with BuAc/EtAc $(1: 1, v v)$ at physiological and alkaline $\mathrm{pH}$ turned out to be the best compromise. Due to the high sensitivity of the 5500 QTrap instrument, $20 \mathrm{mg}$ of body tissue or $20 \mu \mathrm{L}$ of body fluid were sufficient to quantitate low therapeutic concentrations for the majority of the analytes even if LLE was not the optimal extraction procedure as, e.g., for morphine.

\section{Calibration optimization}

Usually, it is recommended to perform calibration in the matrix type of the analyzed sample. Due to the high number of investigated matrices in one case and the fact that blank postmortem tissue samples are difficult to obtain in sufficient quantity, it was impossible to generate calibration curves for all 11 matrices. For practical reasons, a simple solvent calibration was chosen for all matrices. It has to be mentioned that the major focus of the presented method should be the precise comparison between different time points in one sample matrix instead of high accuracy between different matrices. Therefore, although being aware of the fact that a solvent calibration may lead to accuracy problems, it was a reasonable approach to compare concentration changes within one type of matrix.

Another major challenge in quantifying so many compounds with different therapeutic and/or toxic concentration ranges in different matrices in only one analytical run is to find calibration models which can handle the huge dynamic range without source or detector saturation of the MS. Some neuroleptics such as flupentixol have therapeutic blood concentration ranges in the low microgram-per-liter range while, e.g., trazodone has therapeutic blood levels up to $2500 \mu \mathrm{g} / \mathrm{L}$. Starting the analysis, it is usually unknown whether 
therapeutic or even toxic levels several times higher than expected from recommended therapeutic blood concentrations are present. Furthermore, large concentration differences can occur between the different matrices. CSF contained mostly very low concentrations, whereas, e.g., liver and lung usually contained concentrations up to 100 to 200 times higher than those in the blood $[2,20,23]$. As biopsy samples could be analyzed only once, the calibration range had to cover an extreme dynamic range. Trying to calibrate in such a wide range led to MS saturation problems. In a first step, alternative MRM transitions with lower intensities were chosen as quantifiers. Additionally, the declustering potentials were modified to be in a less optimal range, to further lower the signal intensities. However, although the use of alternative MRM transitions and modification of the declustering potentials lead to less saturation in higher concentrations, low therapeutic concentrations could not be analyzed anymore. In a second approach, a third MRM transition was introduced corresponding to the ${ }^{13} \mathrm{C}$ isotope of MRM 1 . As ${ }^{13} \mathrm{C}$ makes up only about $1.1 \%$ of natural carbon, this signal was expected to be less intense and less prone to saturation allowing a linear calibration even in highest concentrations. As exemplarily shown for risperidone in Fig. 1, the sole use of ${ }^{12} \mathrm{C}$ calibration showed strong saturation effects at higher concentrations (A), while dividing the whole calibration range in MRM $1\left({ }^{12} \mathrm{C}\right)$ for lower (B) and MRM $3\left({ }^{13} \mathrm{C}\right)$ for higher concentrations (C) resulted in linear calibration models over the whole respective range. In mass spectrometry, ${ }^{13} \mathrm{C}$ isotopes have various applications. They are used to label target molecules for example in metabolomics or proteomics [24] or are used to produce labeled internal standards [25]. Naturally and unnaturally occurring ${ }^{12} \mathrm{C} /{ }^{13} \mathrm{C}$ ratios are determined in environmental, food, medicinal, or forensic chemistry using isotope ratio mass spectrometry [26]. The general validity of this calibration approach was further investigated and proven during the final validation process as described below.

\section{LC-MS/MS optimization}

The final MRM chromatogram of the 83 analytes (MRM 1) is given in Fig. 2. The presence of the 83 analytes was successfully screened for by mass spectrometry with selected MRM for each analyte using two MRM transitions for ${ }^{12} \mathrm{C}$ and an additional MRM 3 for ${ }^{13} \mathrm{C}$. Only norfluoxetine showed only one fragment in MRM 1 and 3. Generally, guidelines recommend the use of two qualifying ion fragments in addition to the quantifier. In the presented method, MRM 3 was exclusively a quantifier. The addition of another qualifier ion would have led to increased cycle times and consequently to a loss of data points. In real cases, analyte identity is already confirmed prior to quantitation, as heart blood is screened using a different LC-MS/MS screening method including enhanced product ion scans and comparison of the mass spectra with an in- house library. Therefore, monitoring of one qualifier should be sufficient in the quantitation method. Even monitoring of over 80 compounds with three MRM transitions each was only possible using a new, advanced scheduled MRM mode in Analyst ${ }^{\circledR}$ 1.6.2 software. After introducing a certain intensity threshold for each analyte, MRM 2 and 3 were only measured as long as MRM 1 stayed above the defined intensity threshold. Additionally, the scheduled MRM windows were narrowed for the later part of the chromatogram where many analytes were co-eluting. Combining intensity thresholds for each analyte and variable scheduled MRM windows, a minimum of 8 to 10 data points per peak was achieved for the majority of the analytes. As expected, baseline separation of over 80 analytes is impossible within reasonable runtimes. Especially in the last $2 \mathrm{~min}$, several analytes are still coeluting but could be sufficiently differentiated by mass spectrometry with exception of fluoxetine/methadone and ${ }^{13} \mathrm{C}$-clomipramine/chlorprothixene. Some analyte pairs like codeined $3 /{ }^{13} \mathrm{C}$-dihydrocodeine and venlafaxine-d6/7aminoflunitrazepam were overlapping only at high concentrations.

Besides interference in MRM transitions possibly leading to wrong identification, co-elution of two or more compounds might result in ion suppression or ion enhancement between various analytes, especially when using ESI. Such effects, when present in the analyte mixture of calibration samples, but not in authentic samples would result in falsely increased or decreased quantitation results [27]. However, as comparison of analyte concentrations at different time points in the very same case would still be possible in a relative manner, ion suppression and enhancement of co-eluting analytes should play a minor role and was therefore not further investigated.

\section{Method validation}

Validation was performed based on national and international guidelines $[16,28]$ with some modifications as described in the respective sections. Validation of a huge number of 83 analytes in 11 different matrices required these modifications and since special focus for a future PMR study was on an acceptable intra-day precision, some enlargements of acceptance limits for other parameters were acceptable. Even then, it is clear that criteria could not be met for all analytes in every respective matrix. Still, overall results of validation were surprisingly good.

\section{Selectivity and crosstalk}

No interfering peaks were detected in the blank matrices of $\mathrm{FB}, \mathrm{HB}$, and CSF. Peaks at the retention time of diazepam (MRM 1-3), EME (MRM 1), MPH (MRM 1), tramadol (MRM 1), and venlafaxine (MRM 1) were detected in some of the blank tissue matrices but in negligible amounts below 
Fig. 1 Risperidone calibration over the whole concentration range showing strong saturation effects in higher concentrations (a). Division of the total calibration curve in MRM $1\left({ }^{12} \mathrm{C}\right)$ for lower (b) and MRM $3\left({ }^{13} \mathrm{C}\right)$ for higher concentrations (c) resulted in linear calibration models over the whole concentration range. QC low was only calculated using ${ }^{12} \mathrm{C}$ calibration, QC $13 \mathrm{C}$ only using ${ }^{13} \mathrm{C}$ calibration. QC high could be calculated with both calibrations a

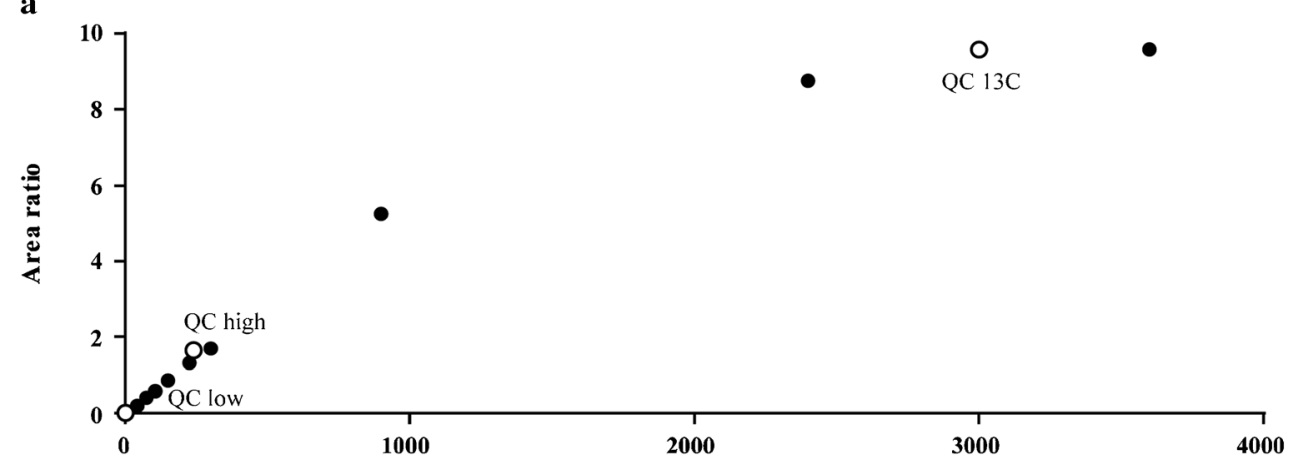

b

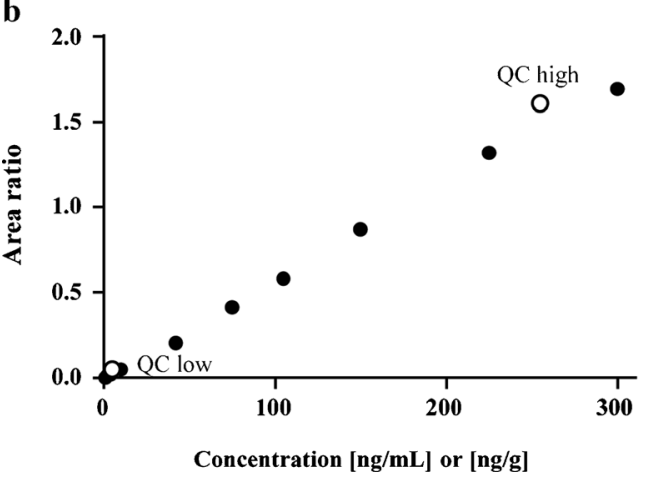

C

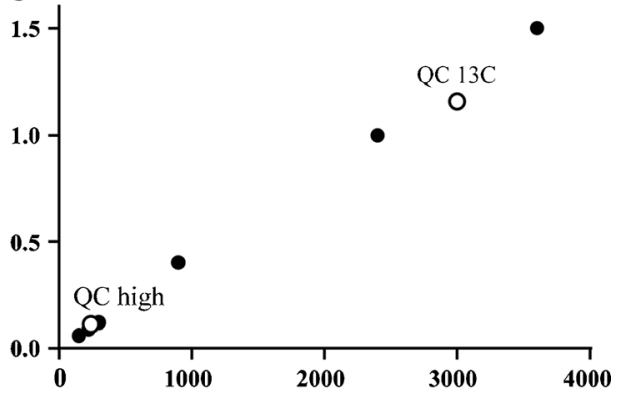

Concentration $[\mathrm{ng} / \mathrm{mL}]$ or $[\mathrm{ng} / \mathrm{g}]$

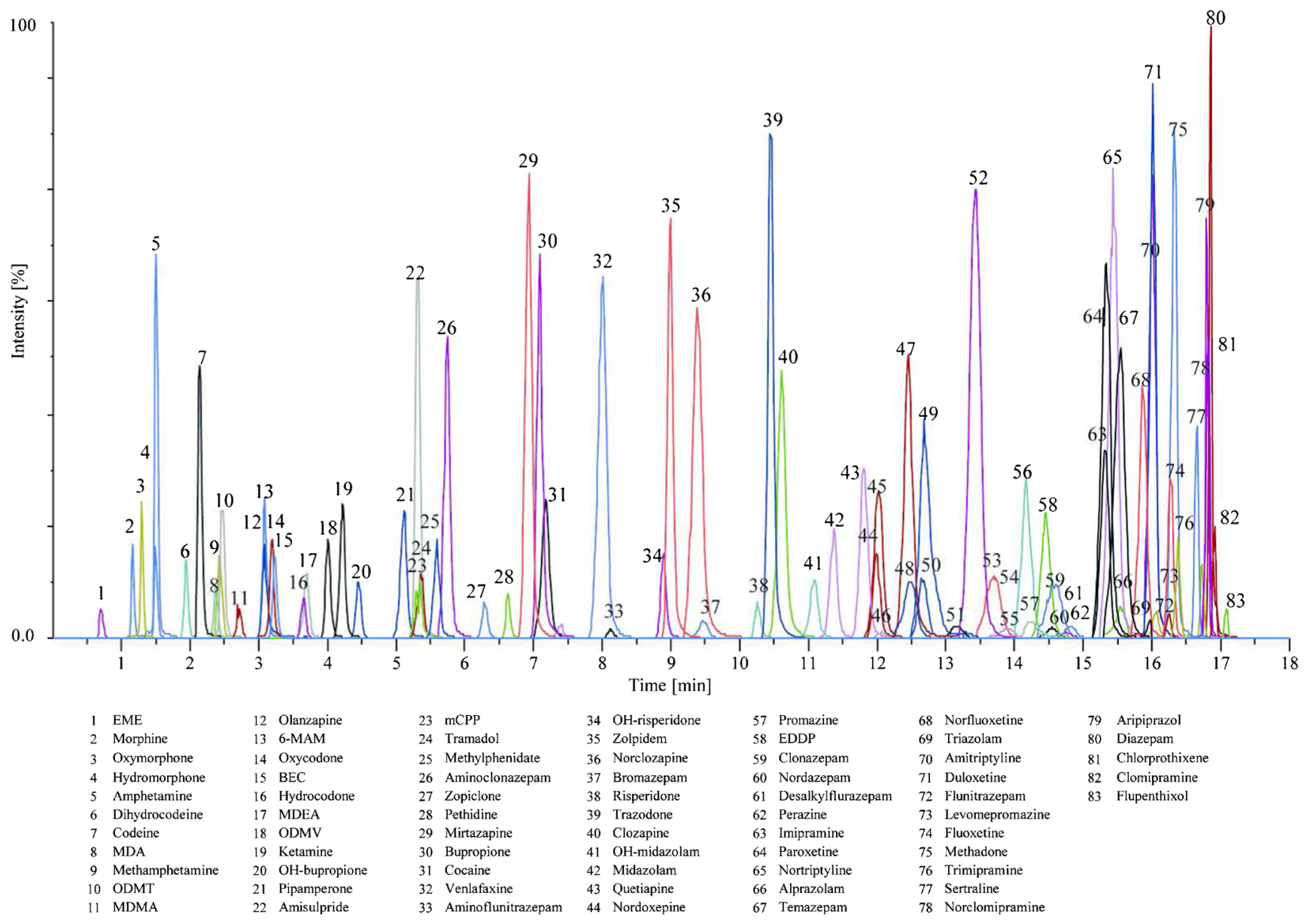

Fig. 2 LC-MS/MS chromatogram of the 83 analytes (MRM 1) 
the lowest calibrator. The IS mixture showed small peaks for clomipramine, norclomipramine, EDDP, HC, MPH, mirtazapine and pethidine, which all had areas far below calibrator 1. However, it has to be mentioned, that all matrices used for this experiment were from authentic cases and had been assigned as "blank" samples based on a routinely performed blood/urine screening. Very low concentrations of some analytes irrelevant for the particular case might have been present and might be the reason for the observed interferences.

Due to the extremely broad calibration range, injection of some analytes at their QC ${ }^{13} \mathrm{C}$ concentrations lead to peak broadening and deterioration of separation from adjacent compounds. For example, DHC and morphine influenced codeine-d3, oxymorphone, and HM. However, the interference peak area of codeine-d 3 made up only $2 \%$ of its regular IS area and was therefore considered as irrelevant. Oxymorphone and HM were only partly influenced and therefore insignificant for routine analysis. 7-aminoflunitrazepam was not baseline separated from venlafaxine-d6 anymore, but comparing the IS areas of the low and high calibrators, the area of venlafaxine-d6 was not influenced to a significant extent. Clomipramine lead to interfering peaks with the quantifier and qualifier of chlorprothixene and norclomipramine at a concentration between calibrators 2 and 3. Injection of clomipramine at its QC high concentration did show the same effect, but concentrations were below the lowest calibrator. On the other hand, chlorprothixene or norclomipramine did not influence clomipramine. As the interferences only appeared in the highest calibrators and the resulting change in peak areas was only small, the accuracy of the calibrators should not be affected to a relevant extent. Problems would arise, if a sample contained high clomipramine concentrations together with low chlorprothixene or norclomipramine concentrations. In such a case, the chlorprothixene and norclomipramine concentrations would have to be interpreted with caution or a separate calibration for each analyte had been necessary. Taking into consideration that such a combination of analytes should be very seldom, results for selectivity and crosstalk were vastly satisfying and in addition, crosstalk was not observed for all other compounds, even in the highest concentrations tested.

\section{Calibration model}

If possible, the simplest linear regression model with $1 / x$ or $1 / x^{2}$ weighting was used. Only analytes that did not fulfill the required criteria using linear regression models were additionally tested for quadratic regression models. The final regression models, weighting factors, and the respective ISs are given in ESM Table S3. QC low was only calculated using ${ }^{12} \mathrm{C}$ calibration, while QC ${ }^{13} \mathrm{C}$ was only calculated using ${ }^{13} \mathrm{C}$ calibration. QC high accuracy was calculated using both calibrations. From the 83 analytes, in the ${ }^{12} \mathrm{C}$ calibration only ten analytes and in the ${ }^{13} \mathrm{C}$ calibration only five analytes did not fulfill the criteria for linear calibration and were therefore fitted to a second-order model to finally meet the criteria. In total, five analytes did not fulfill a back-calculated calibrator concentration of $\pm 30 \%$ in the ${ }^{12} \mathrm{C}$ calibration and the ${ }^{13} \mathrm{C}$ calibration in all regression models, what may have been caused by the lack of an appropriate IS.

\section{Matrix effects and extraction efficiency}

Based on international validation guidelines, EE and ME studies are usually performed at two concentration levels, low and high $[16,28]$. Due to the high number of analytes and different postmortem matrices, the ME and EE experiments were only performed at QC med. ME data and RSD of all analytes in all matrices are summarized in ESM Table S4. Briefly, an ion suppression greater than $25 \%$ in all matrices was observed for EME, norfluoxetine, flupentixol, hydromorphone, oxymorphone, and sertraline, while agomelatine, amitriptyline, nortryptiline, aripiprazole, chlorprothixene, clomipramine, norclomipramine, clonazepam, norclozapine, diazepam, nordazepam, duloxetine, flunitrazepam, fluoxetine, desalkylflurazepam, haloperidol, nitrazepam, paroxetine, and trimipramine exhibited ion suppression only in tissues but not in liquid matrices. Thirty-eight analytes (46\%) were affected by ion suppression exclusively in brain and/or AT. Overall, matrices did not cause ion suppression or enhancement in the same extent. Ion suppression could be observed for only 6 analytes in CSF, for 8 analytes each in FB and HB, and for 12 analytes in urine. CSF was therefore the matrix causing the least ME, followed by blood and urine. Muscle, lung, spleen, and kidney were similarly affected with 22 to 27 suppressed analytes. Worse results could be observed in liver with 36 suppressed analytes and brain and AT with 74 and 80 suppressed analytes, respectively. Brain and AT are the matrices with the highest lipid content, which might be the cause of the extensive ion suppression. The fact that the body fluids showed less ME compared with the solid tissues supports the assumption that lipophilic matrix components are the main cause of ion suppression [29, 30]. Additionally, many analytes which only elute at a higher organic solvent percentage (RT >13 min; where lipophilic matrix is known to elute) were affected by ion suppression in the solid tissues. The ME of EME, morphine, oxymorphone, HM, and amphetamine may be explained by the early elution at an RT less than 2 min that probably falls together with salts from the matrices. In these cases, body fluids and solid tissues were affected to the same extent. Ion enhancement greater than $25 \%$ was observed for 7-aminoclonazepam, 7aminoflunitrazepam, OH-flurazepam, levomepromazine, olanzapine, perazine, pipamperone, promazine, risperidone, and temazepam in different matrices (ESM Table S4). 
Olanzapine is known to be unstable and susceptible to oxidation in biological matrices $[31,32]$. Levomepromazine, perazine, promazine, and promethazine are phenothiazines known to be easily oxidized to sulfoxides [33]. This could be the reason, why these compounds did not pass the specifications. The thioxanthenes chlorprothixene and flupentixol are structurally related to the phenothiazines and might also be affected by oxidation. Interestingly, thioxanthenes were prone to strong ion suppression in contrast to phenothiazines, which suffered from strong ion enhancement. As the thioxanthenes eluted closely together at high retention times, the ion suppression may have been caused by lipophilic matrix components such as fatty acids. As it was expected from postmortem tissues, many compounds exhibited ion suppression or enhancement, but for most analytes these effects were reproducible $(\mathrm{RSD}<15 \%)$ and therefore tolerable, as long as the method was sensitive enough. As given in ESM Table S4, only four analytes in body fluids, eight analytes in muscle, and only one analyte in AT did not pass these criteria. In contrast, 23 to 45 analytes in spleen, lung, kidney, liver, and brain were outside these criteria. Use of an IS - usually applied in routine analysis - could further compensate these deviations in all matrices as shown in summary for all analytes and tissues in Table 1. Briefly, in body fluids only olanzapine was still above the set criteria of $15 \%$ and in body tissues only bupropion, flupentixol, EDDP, olanzapine, opipramol, oxymorphone, perazine, sertraline, ODMT, and ODMV still exceeded the criteria. The mentioned analytes that did not benefit from IS compensation, were analytes without an appropriate IS with comparable structure or RT.

Determination of EE was done comparing blank tissue matrix spiked before and after extraction. Of course, this may not reflect the true recovery of substances from authentic tissue samples, where the substances may not be dissolved to the same extent as after spiking them onto the matrix. However, recovery from authentic samples could not be determined for over 80 analytes in several tissues due to unavailability of sufficient reference material and for practical reasons. Therefore, the approach in the present experiment seemed to be the most appropriate. A summary of the EE for all analytes in all matrices is given in ESM Table S5. Generally, EE was between 70 and $90 \%$ for the majority of the analytes in the different matrices. EE below $50 \%$ in all matrices was only observed for BEC and morphine. In blood, CSF, urine, kidney, muscle, lung, and AT, 3 to 10 analytes, and in liver, brain, and spleen, 16 to 24 analytes had EEs below 50 \%. Especially, considering the fact that a solvent calibration was used, low EE from matrix should result in bad accuracy. However, as already described for ME, usage of IS may have been compensated for bad EE as shown in ESM Table S6. With IS compensation, only nortryptiline, bupropione, norclozapine, flupentixol, levomepromazine, EDDP, nitrazepam, olanzapine, opipramol, oxymorphone, perazine, promazine, promethazine, and sertraline had recoveries below $80 \%$. Standard deviation above $15 \%$ indicating unreproducible $\mathrm{EE}$ in more than one matrix was only seen for nortryptiline, bromazepam, bupropion, norclozapine, nordoxepine, flupentixol, OH-flurazepam, HC, HM, levomepromazine, mCPP, EDDP, olanzapine, opipramol, oxymorphone, perazine, promazine, promethazine, sertraline, ODMT, and ODMV. Overall, ME and EE were comparable with typical multi-analyte methods.

\section{Accuracy and precision}

In forensic toxicology, national and international guidelines exist, which recommend various validation procedures [16, $28]$. If these full validation recommendations exceed the possibilities of a laboratory in terms of time and cost, simplified partial validations can be performed [28]. Due to the high number of matrices, accuracy and precision experiments were performed in a modified validation procedure generating data over 4 instead of 8 days. FB and HB were handled as one matrix named blood in general for accuracy and precision experiments. Results of the accuracy and precision experiments are given in Table 2. As the aim of the future study is to assess concentration differences between 2 and 3 time points - intra-day precision was considered the most important point to focus on in the method validation process. Therefore, bias, intra-day, and inter-day precision will be discussed separately in the following.

As a solvent calibration was used, bias was expected to some extent, e.g., for those analytes with low recoveries and/ or not having an appropriate IS. However, even if the accuracy did not fulfill the acceptance criteria, concentration differences can still be assessed relatively. Nevertheless, 38 analytes fulfilled the criteria of $\pm 30 \%$ in all matrices. The best results were obtained in body fluids, with $75-81 \%$ of the analytes passing the accuracy requirements. The body tissues comparatively caused bias in more analytes with only $66-71 \%$ of the analytes passing the criteria. Compounds with bad accuracy results (detected in more than two matrices) were nortryptiline, bupropion, $\mathrm{OH}$-bupropion, chlorprothixene, clomipramine, norclozapine, cocaine, BEC, EME, codeine, DHC, nordoxepine, flupentixol, haloperidol, HC, HM, levomepromazine, mCPP, MDEA, EDDP, MPH, morphine, olanzapine, oxycodone, oxymorphone, perazine, pipamperone, promazine, promethazine, quetiapine, sertraline, ODMT, and ODMV. Out of the 51 analytes with an appropriate deuterated IS, $78 \%$ showed good accuracy results. Of the 32 analytes without appropriate IS, only $22 \%$ had acceptable accuracy results.

The intra-day precision criteria were fulfilled by the majority of the analytes (Table 2). There was no apparent difference in precision between the matrices observed. In all matrices, 83-93\% of the analytes had an intra-day precision below 


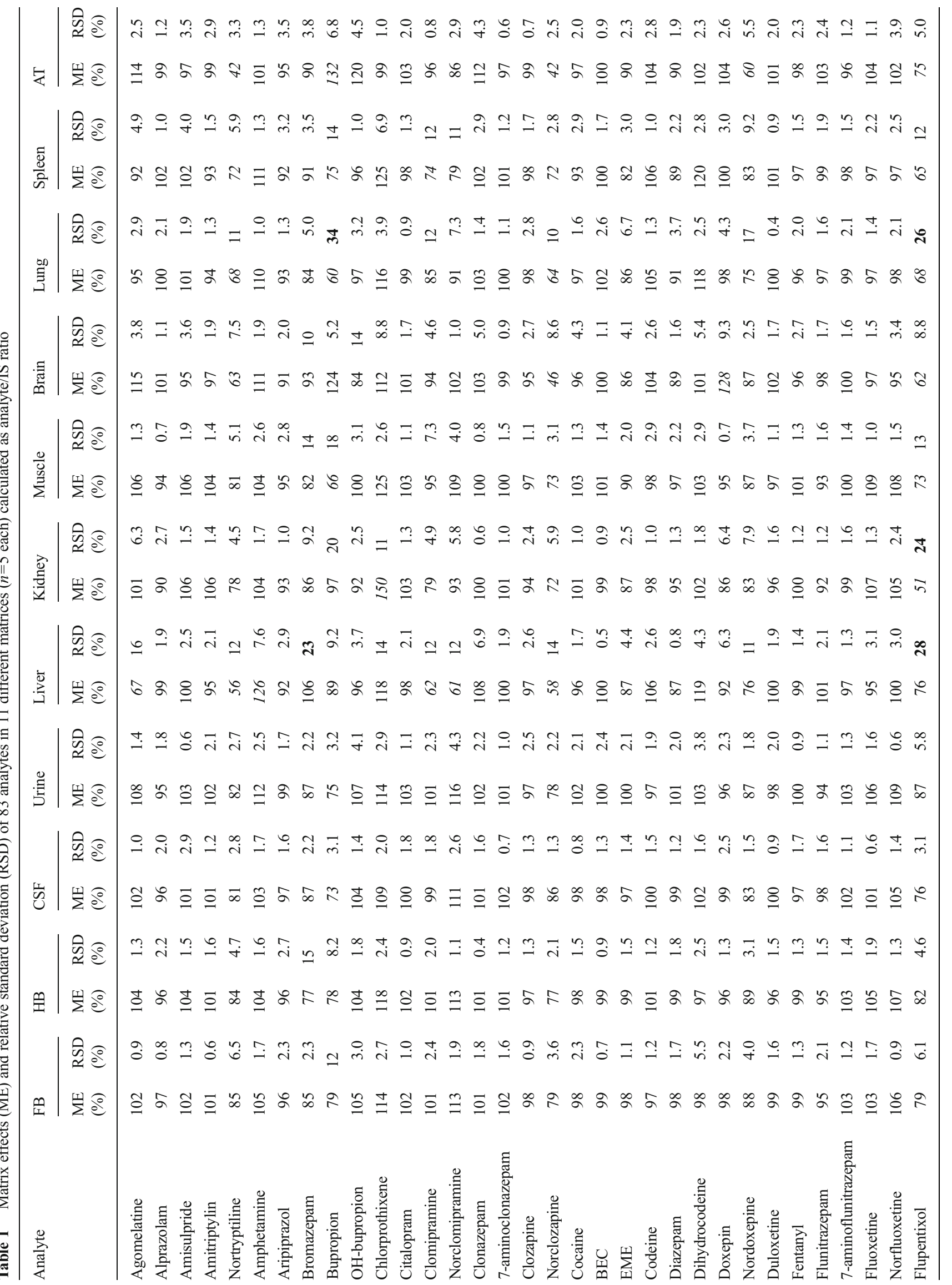




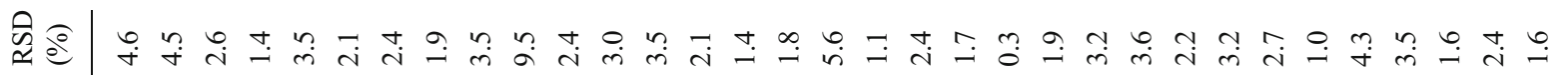

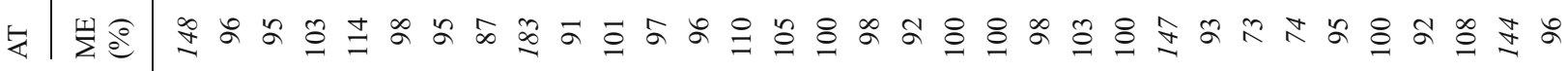

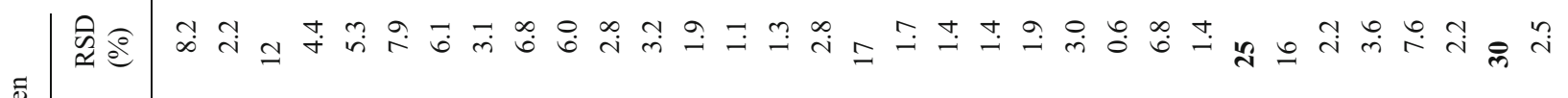
总 ॠ ॠ

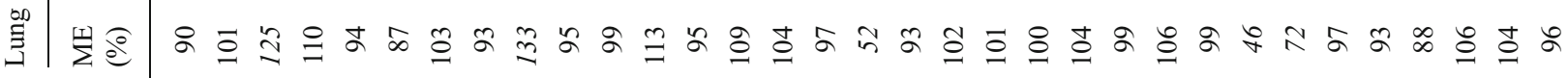
क्षे

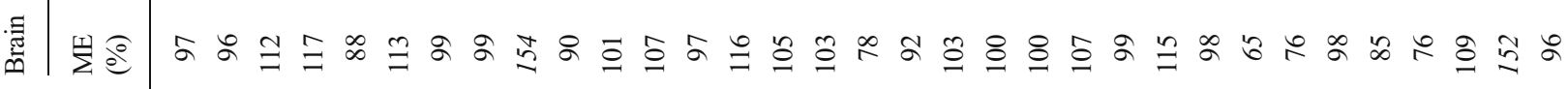

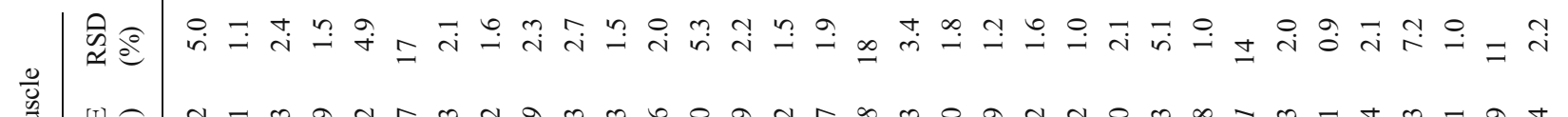

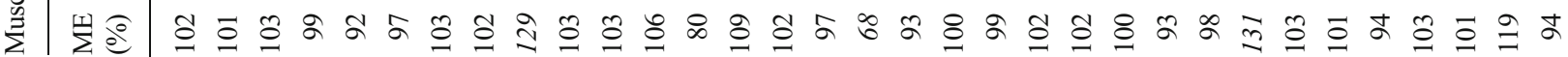

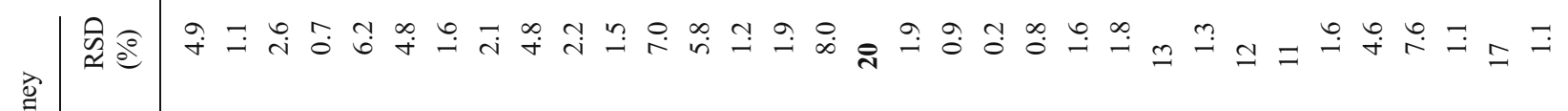

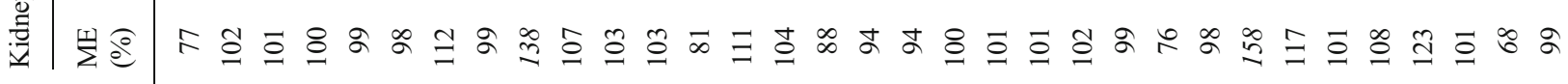

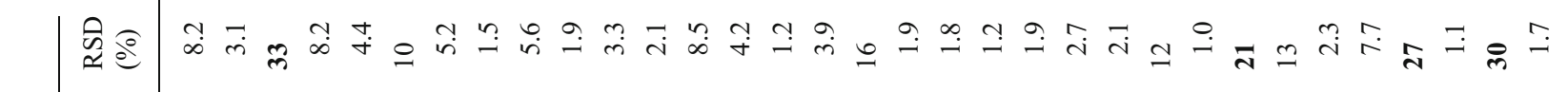

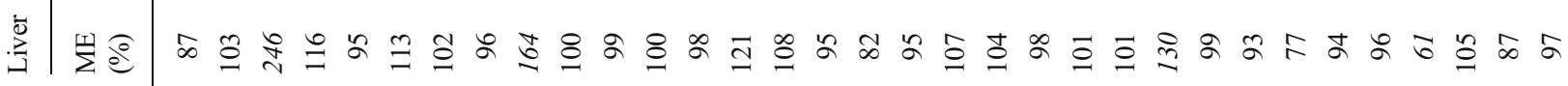

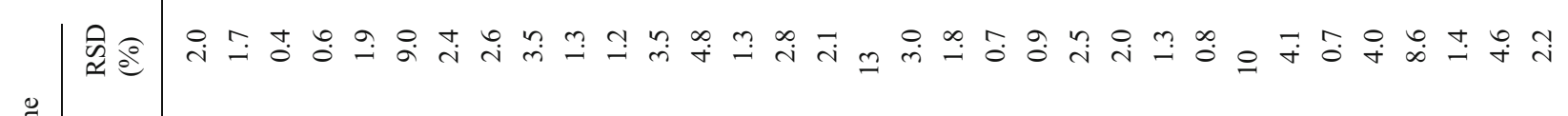

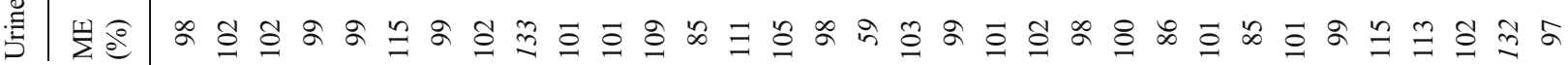

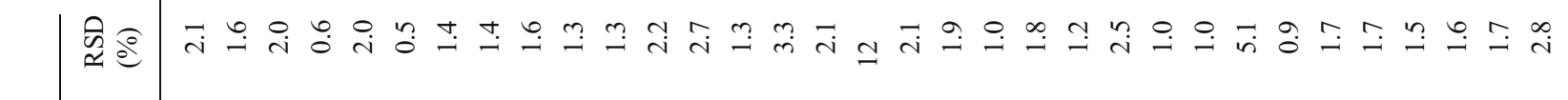

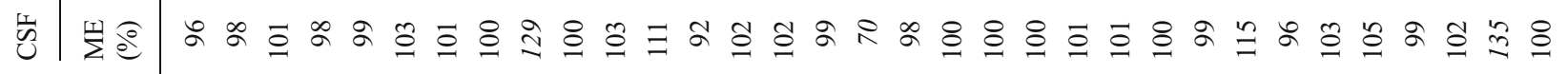

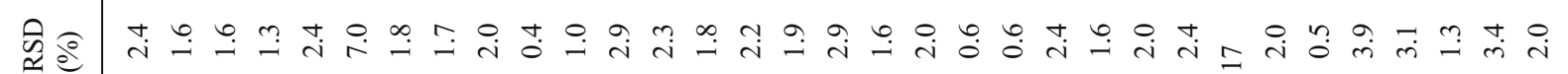

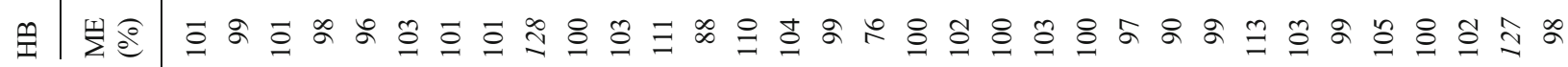

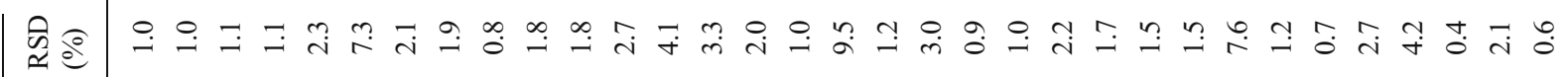

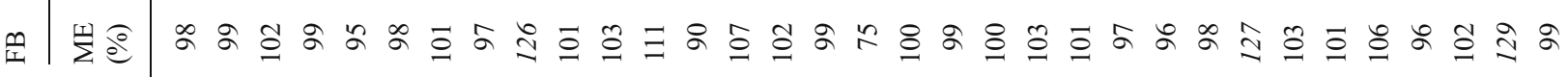




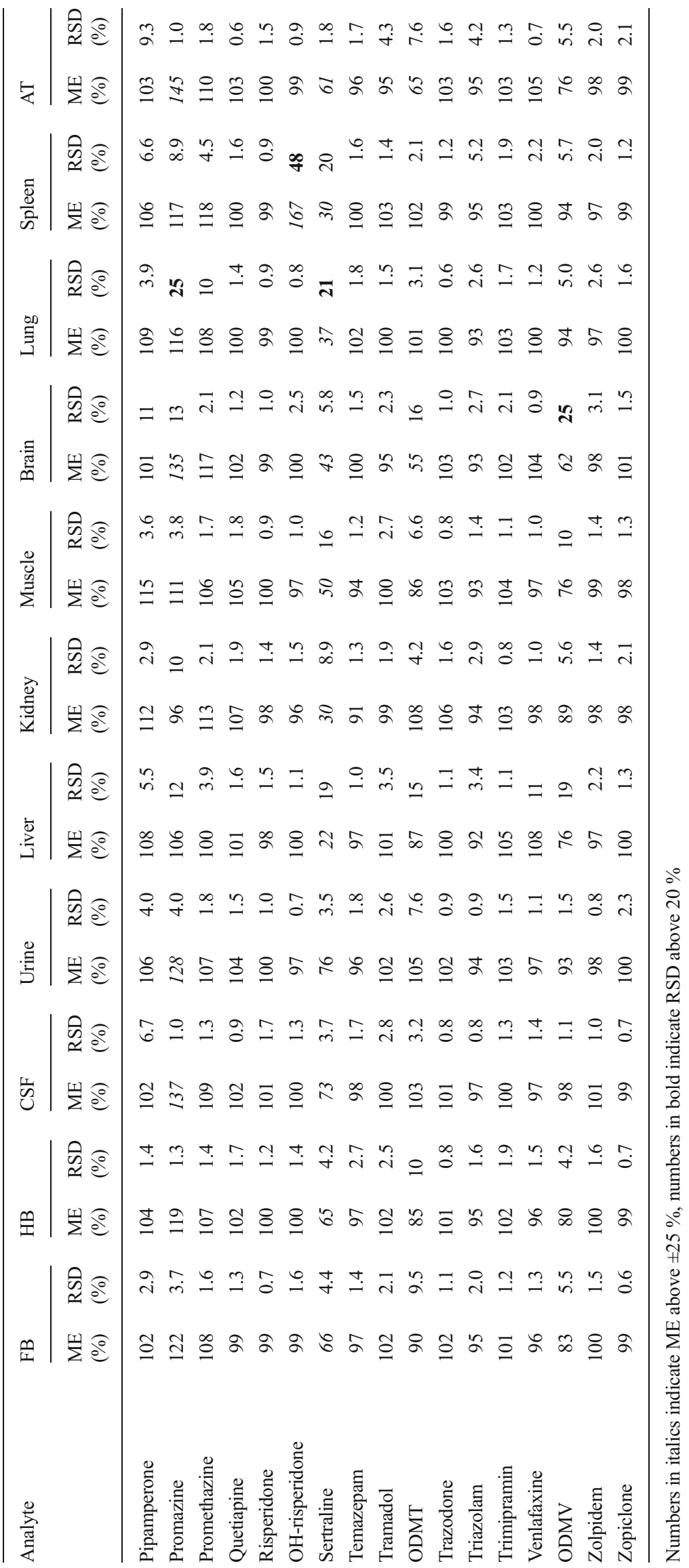




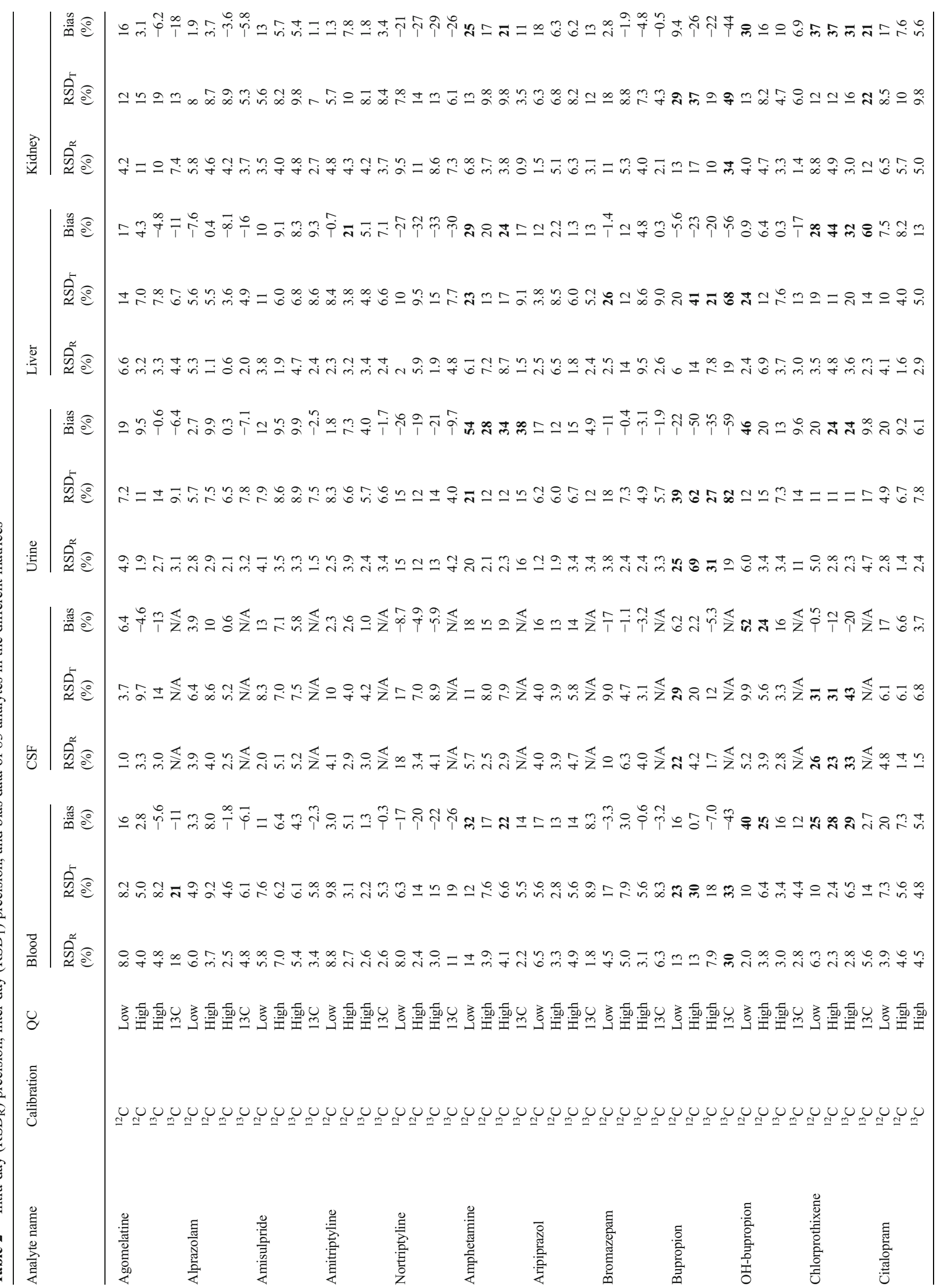




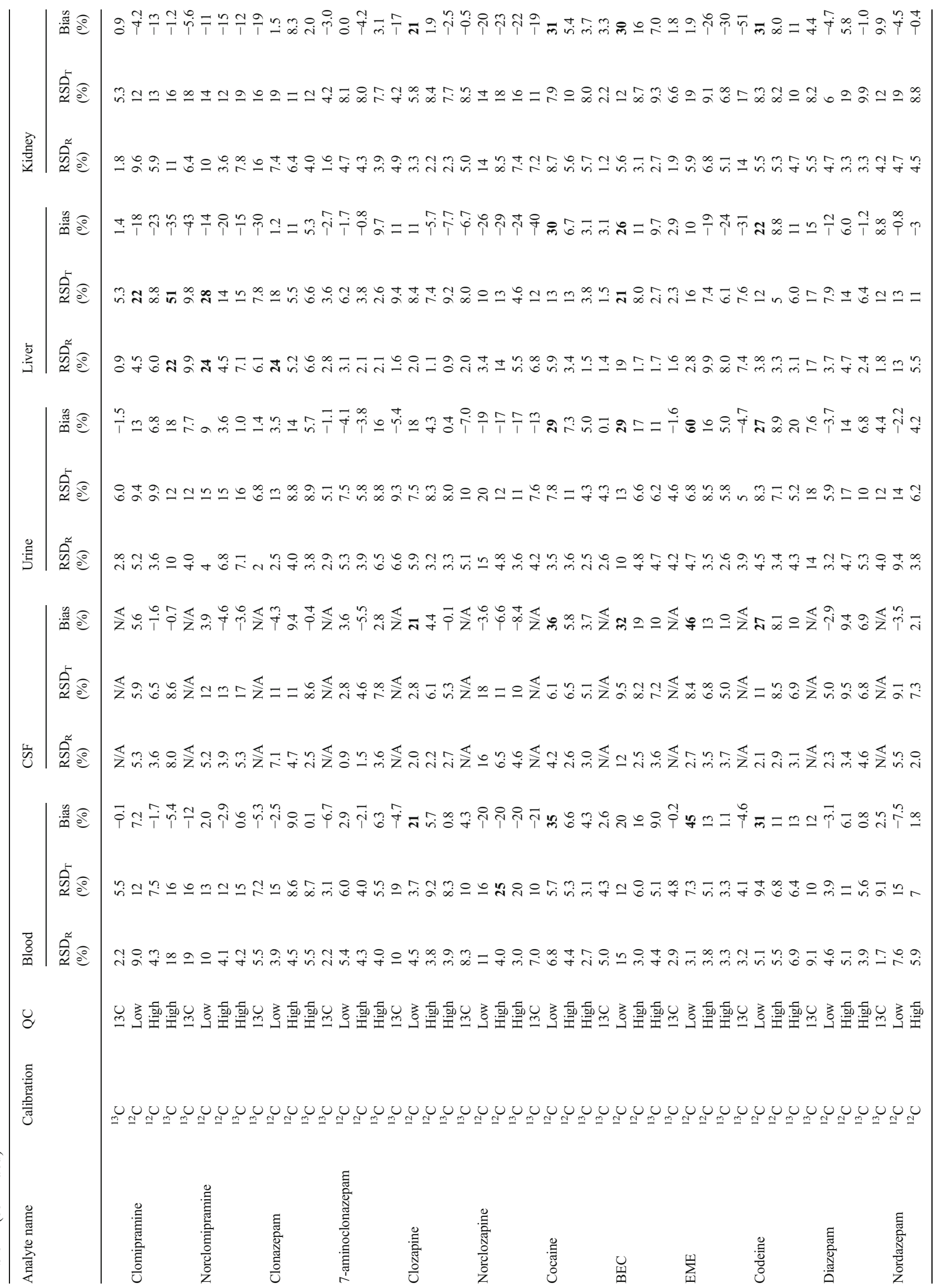




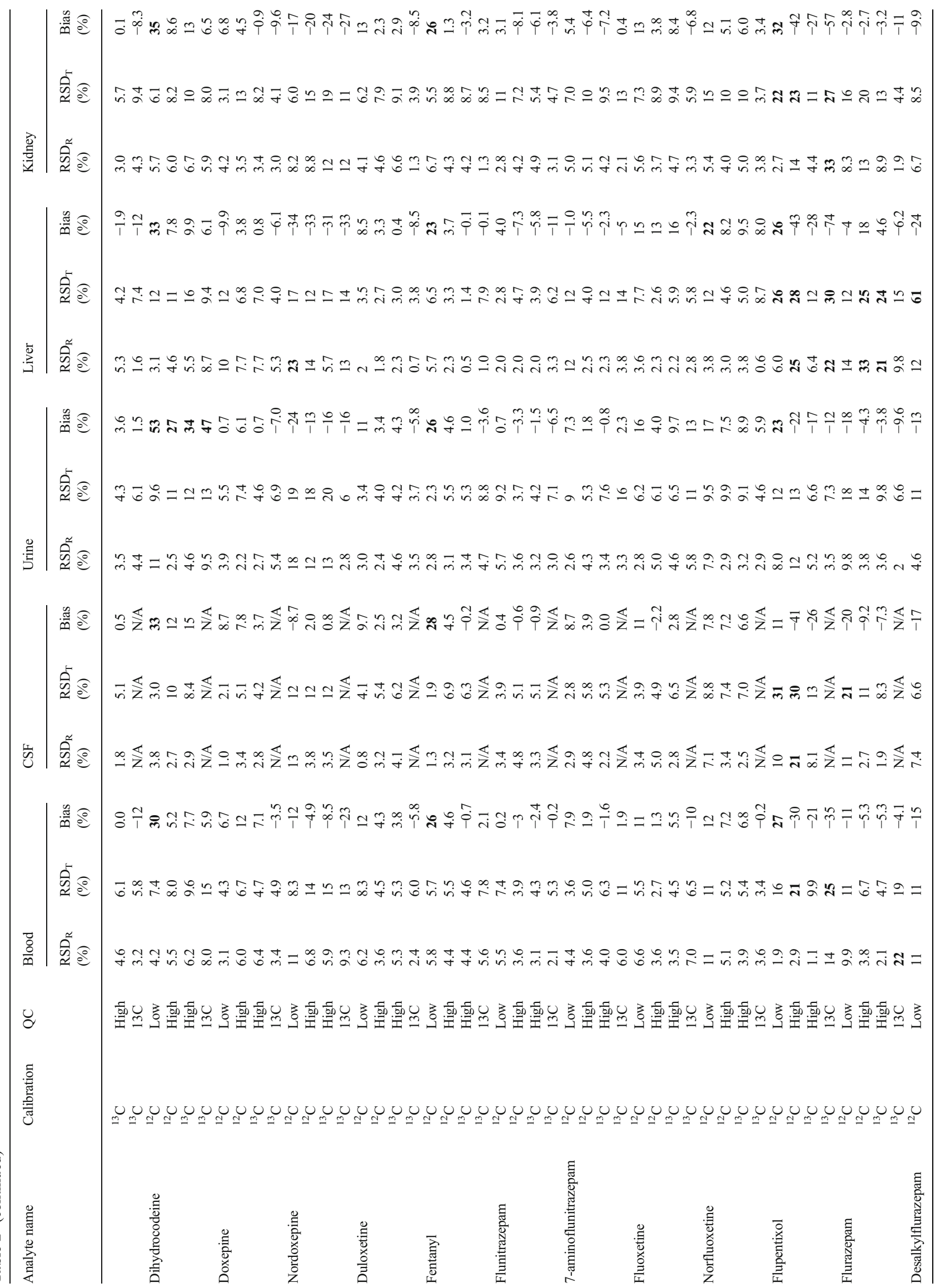




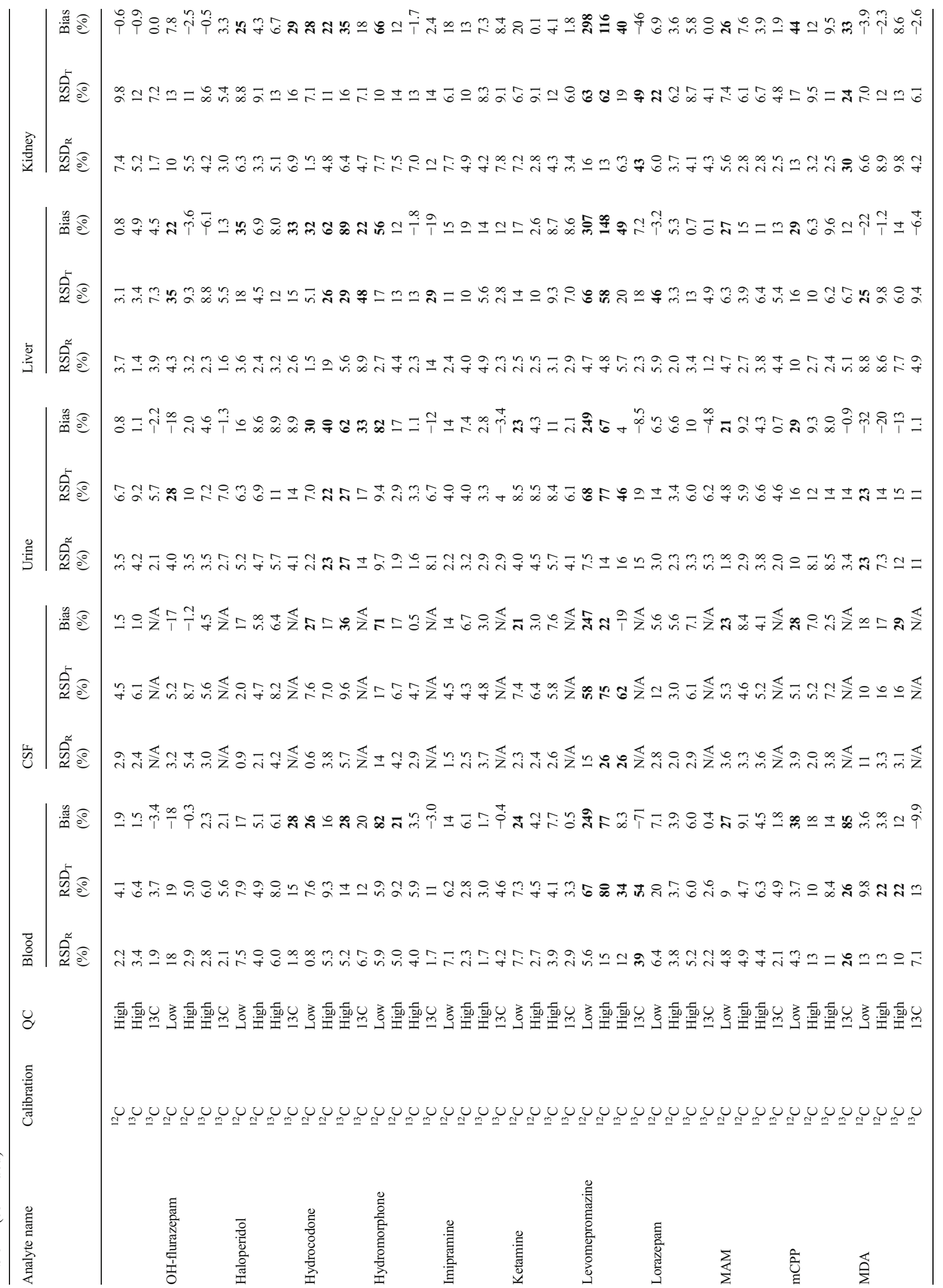




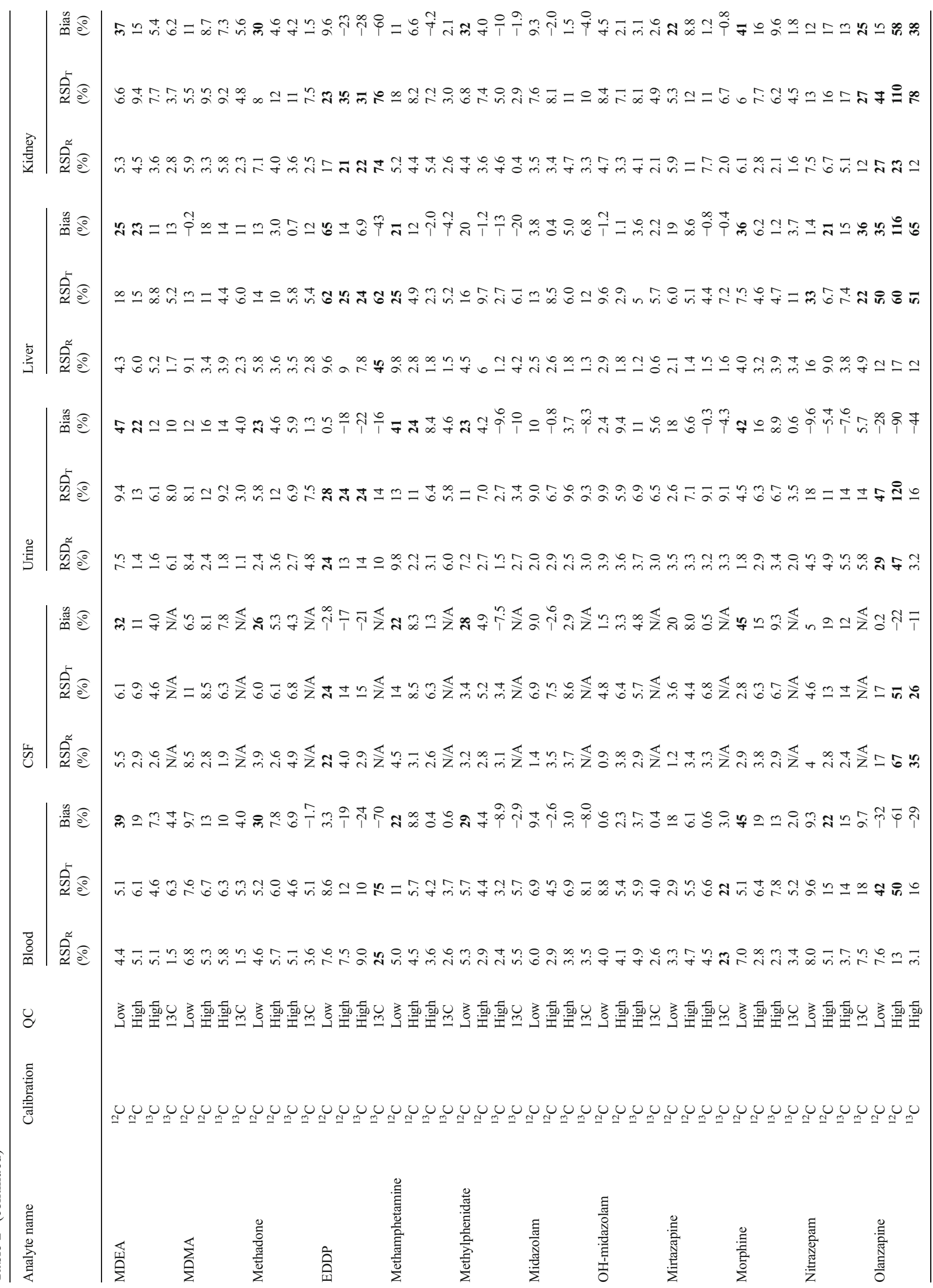




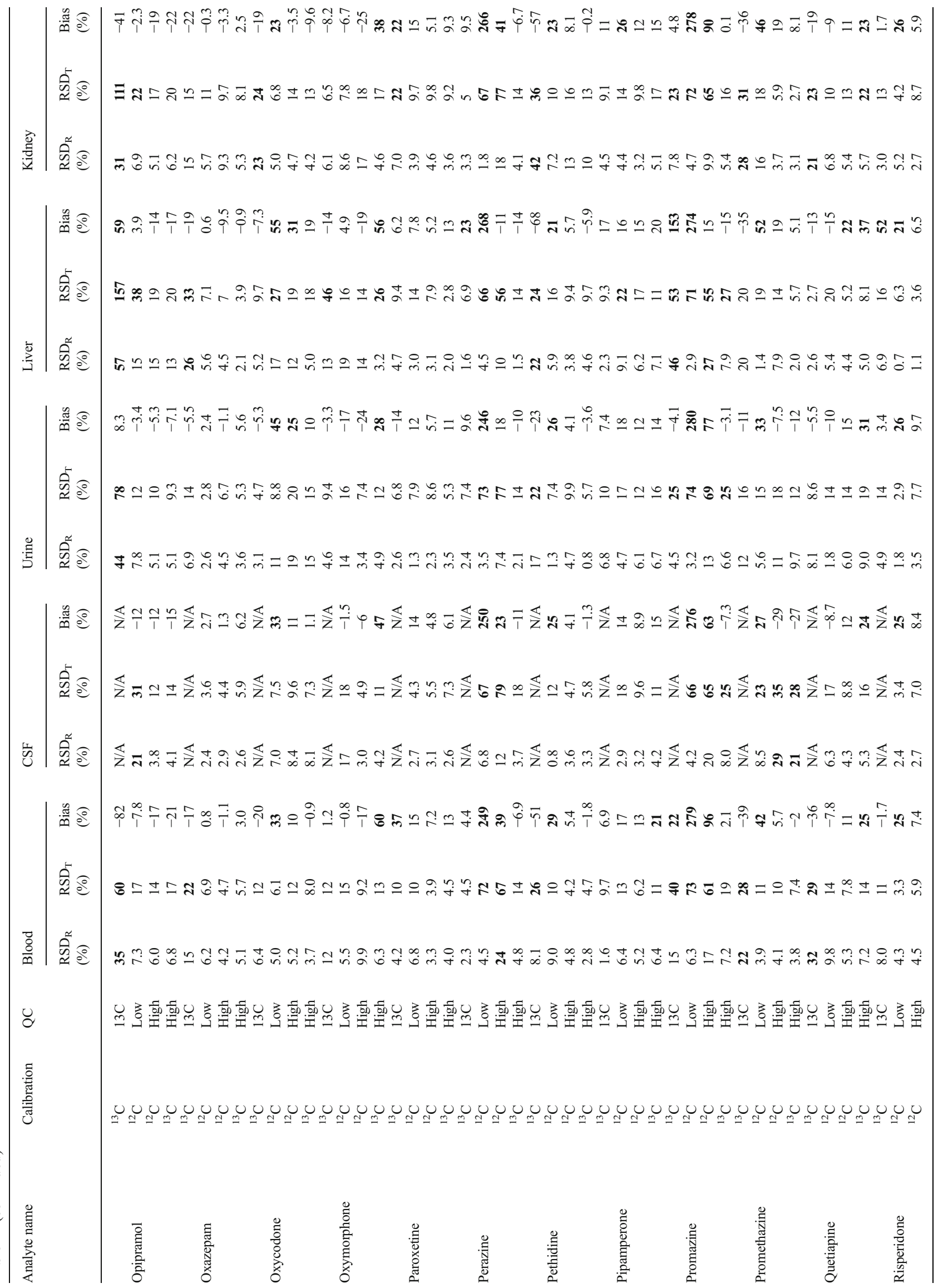




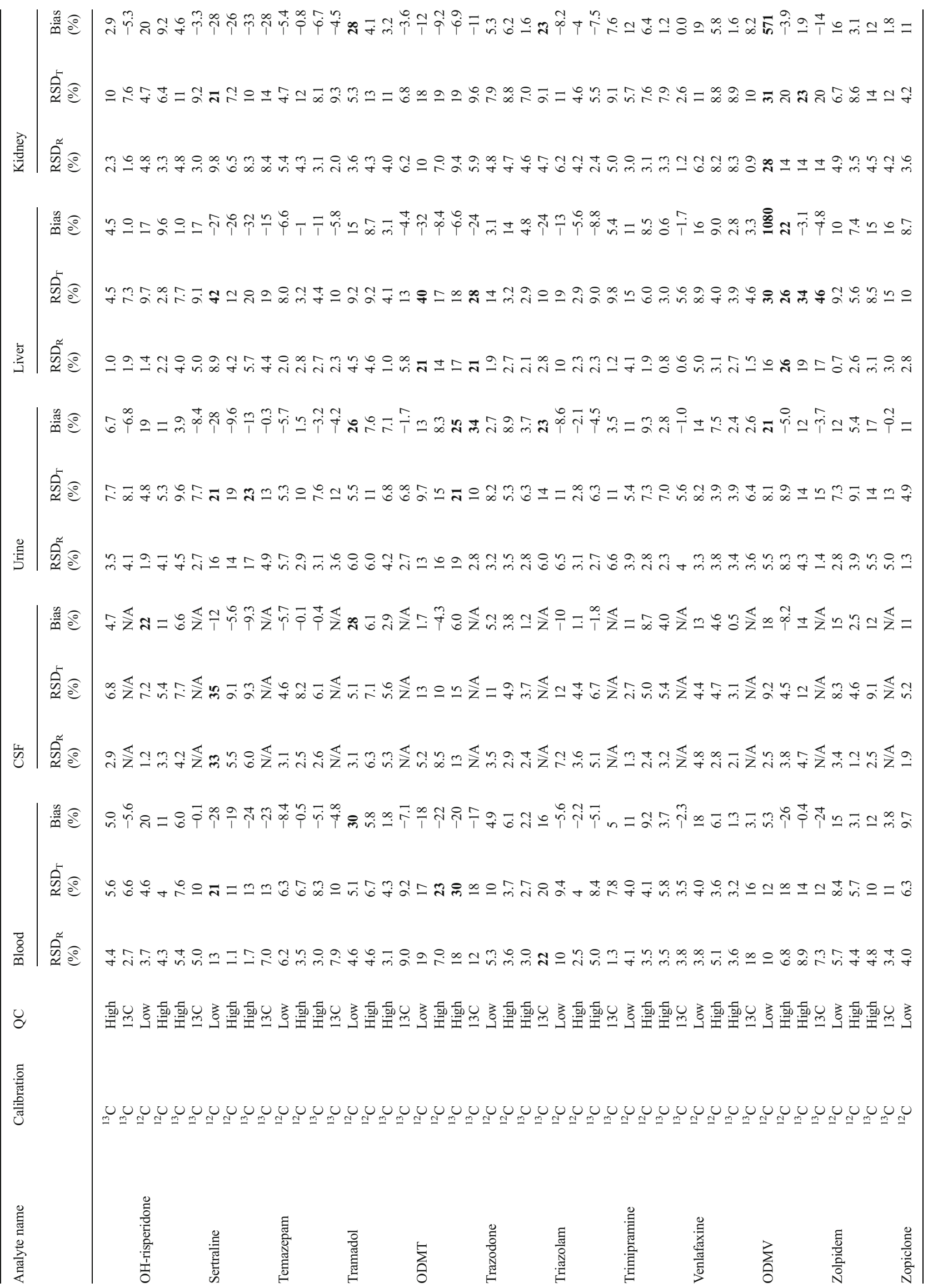




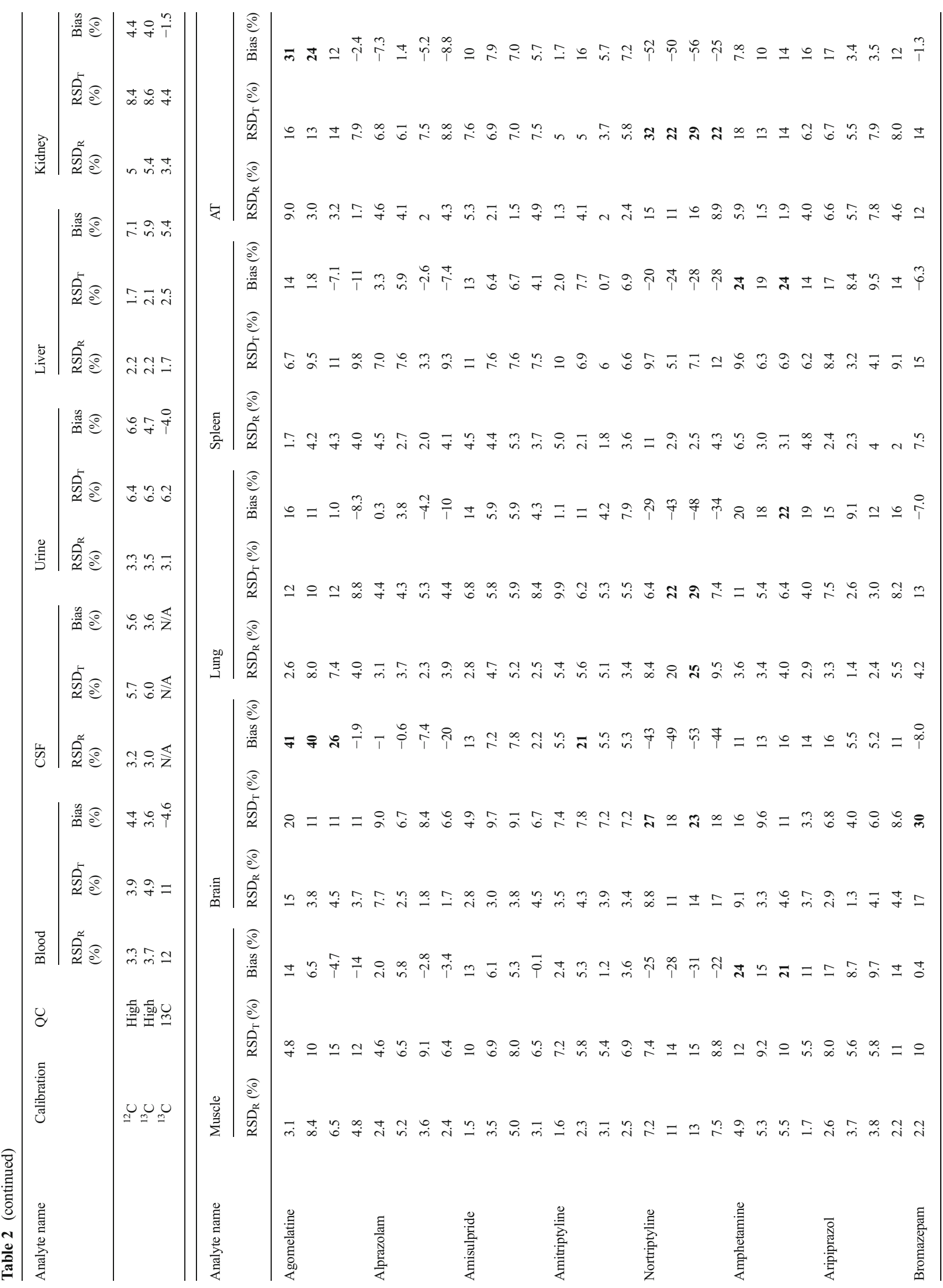




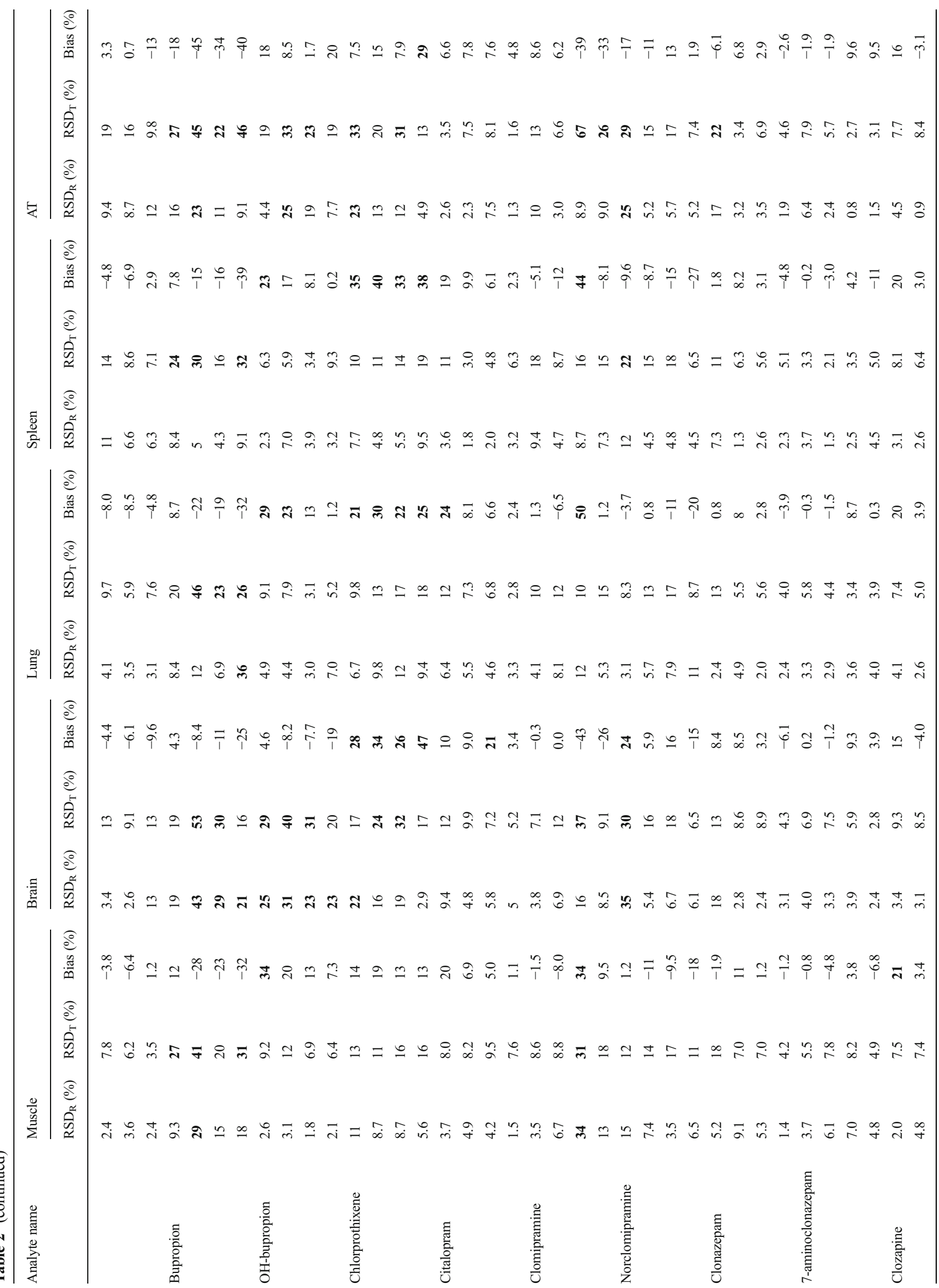




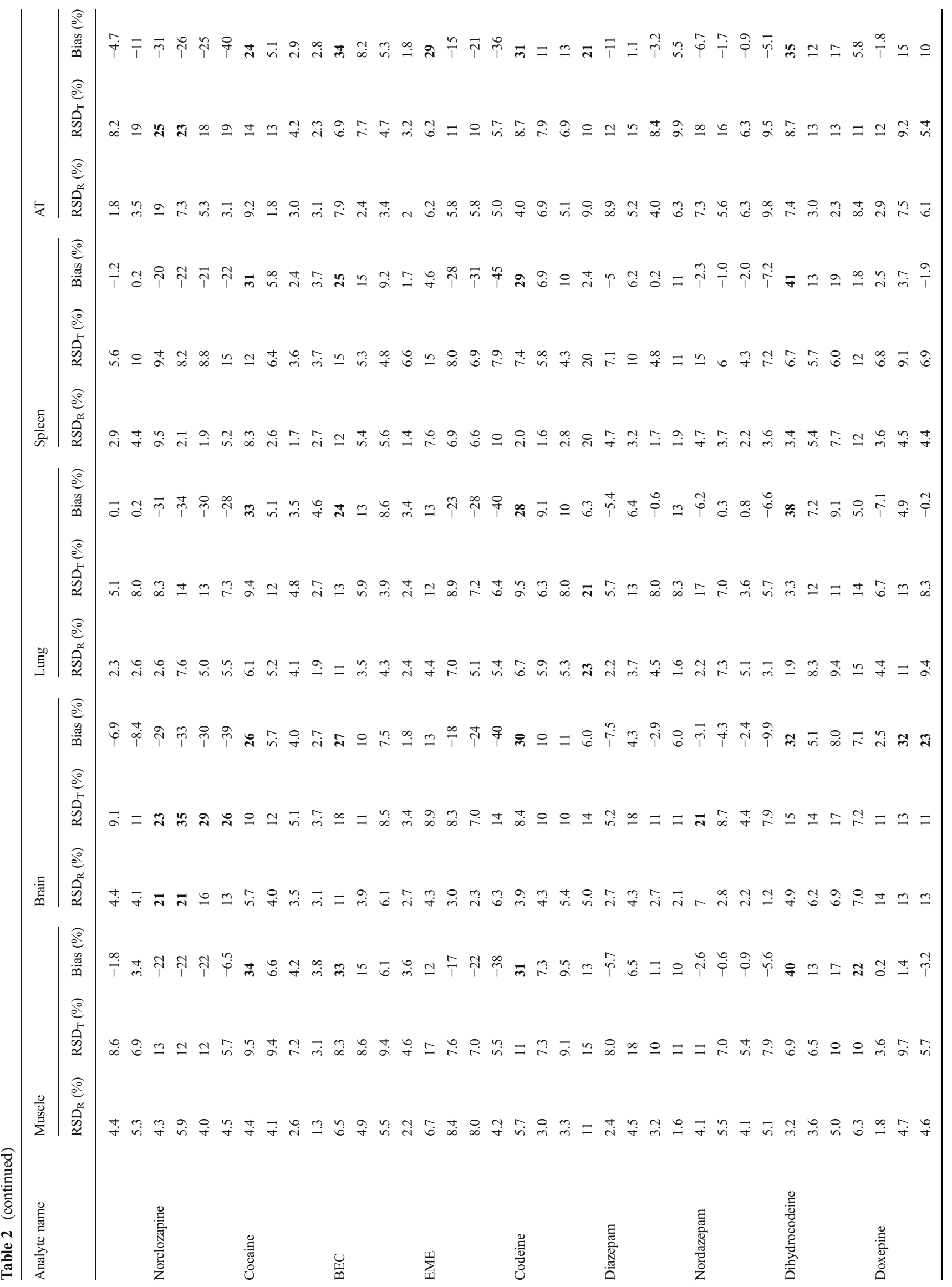




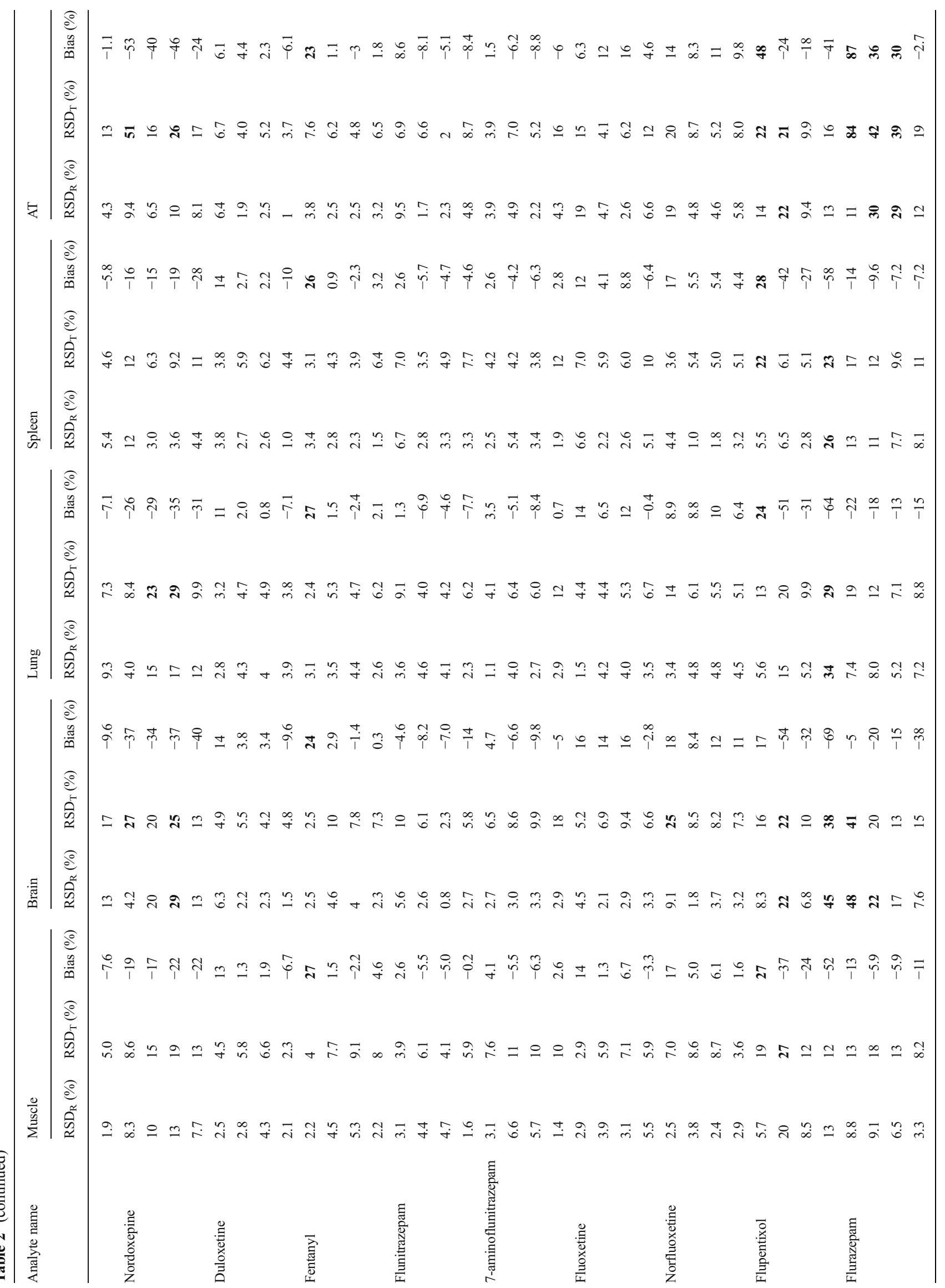




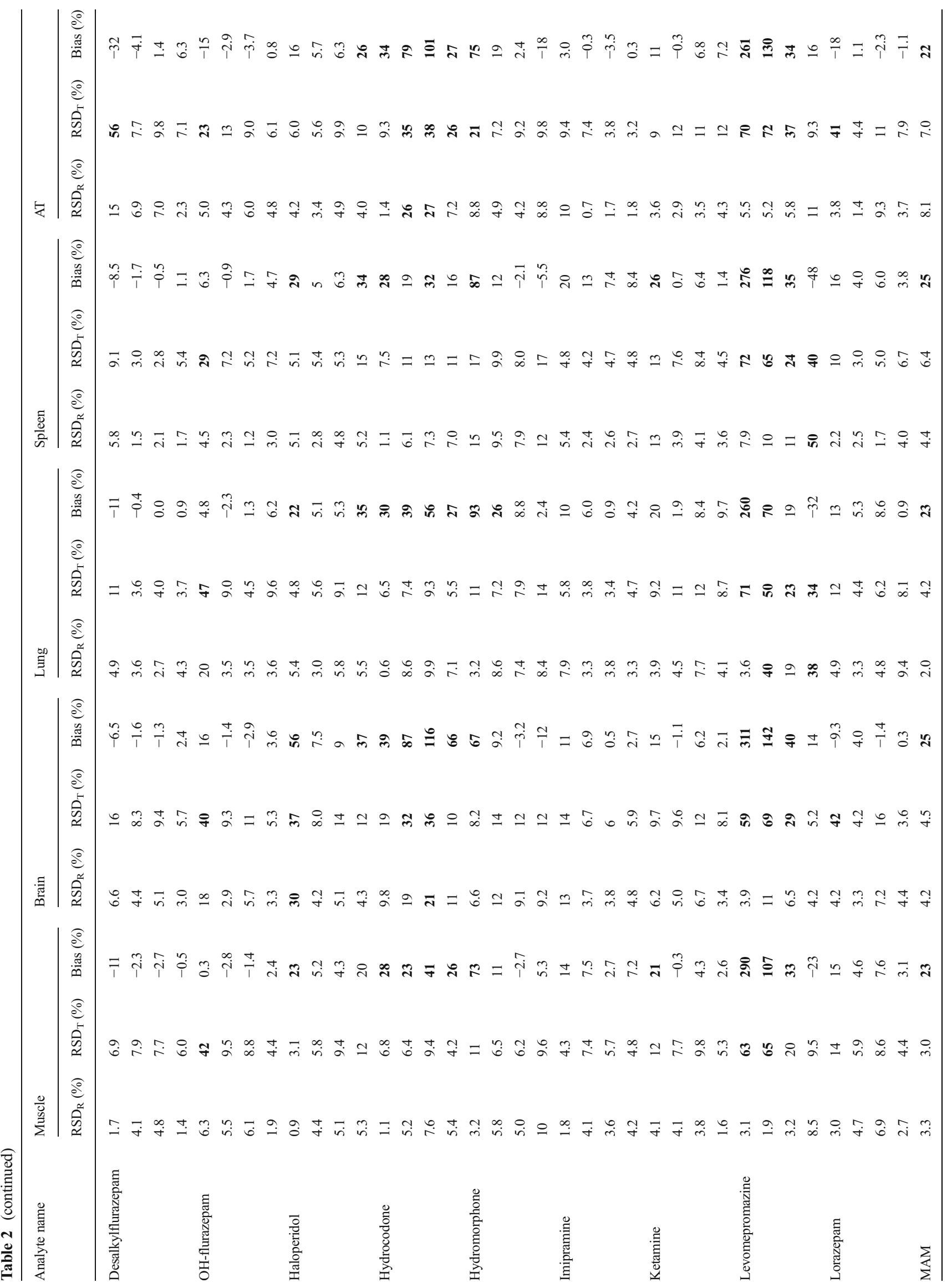




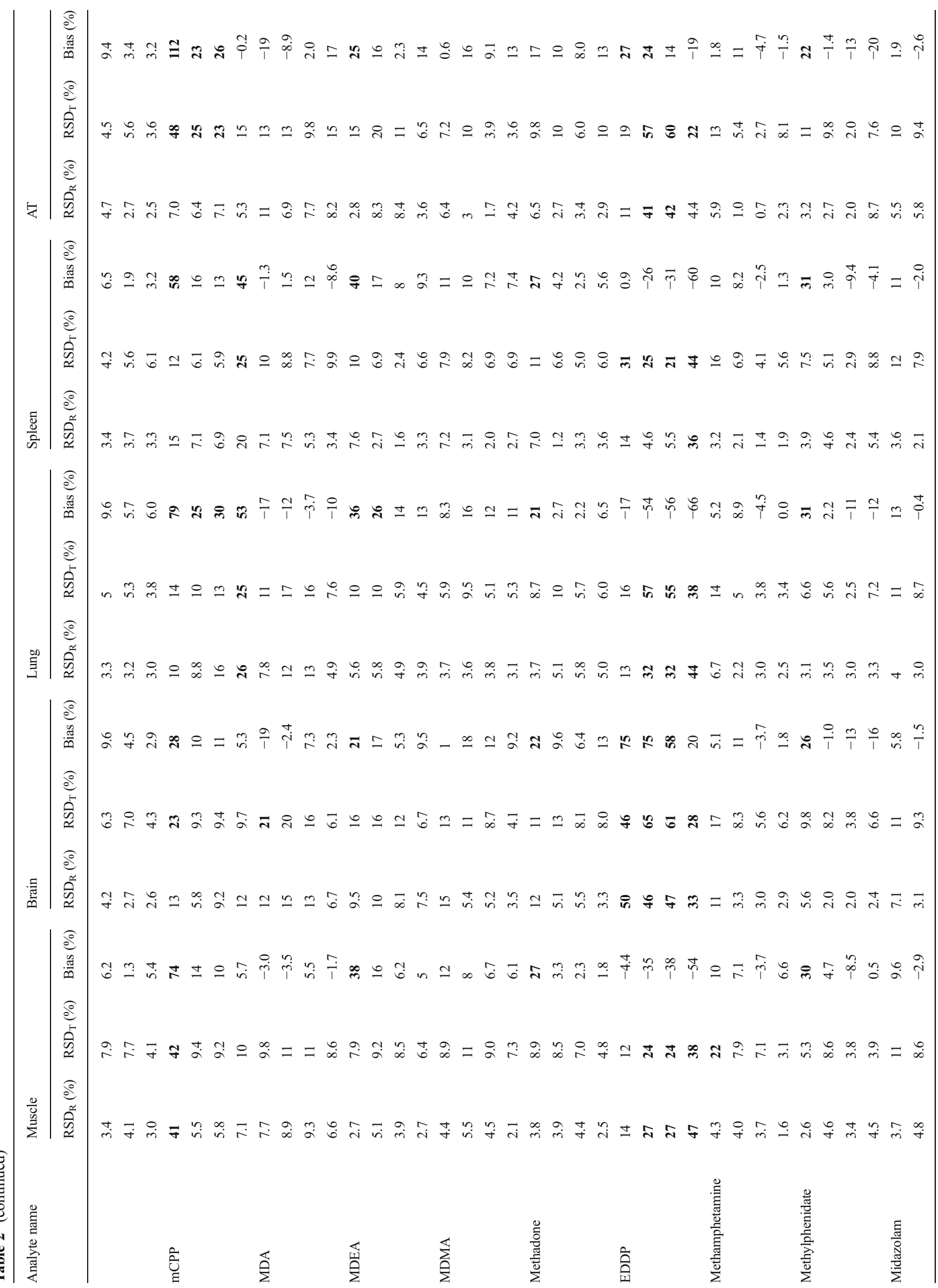




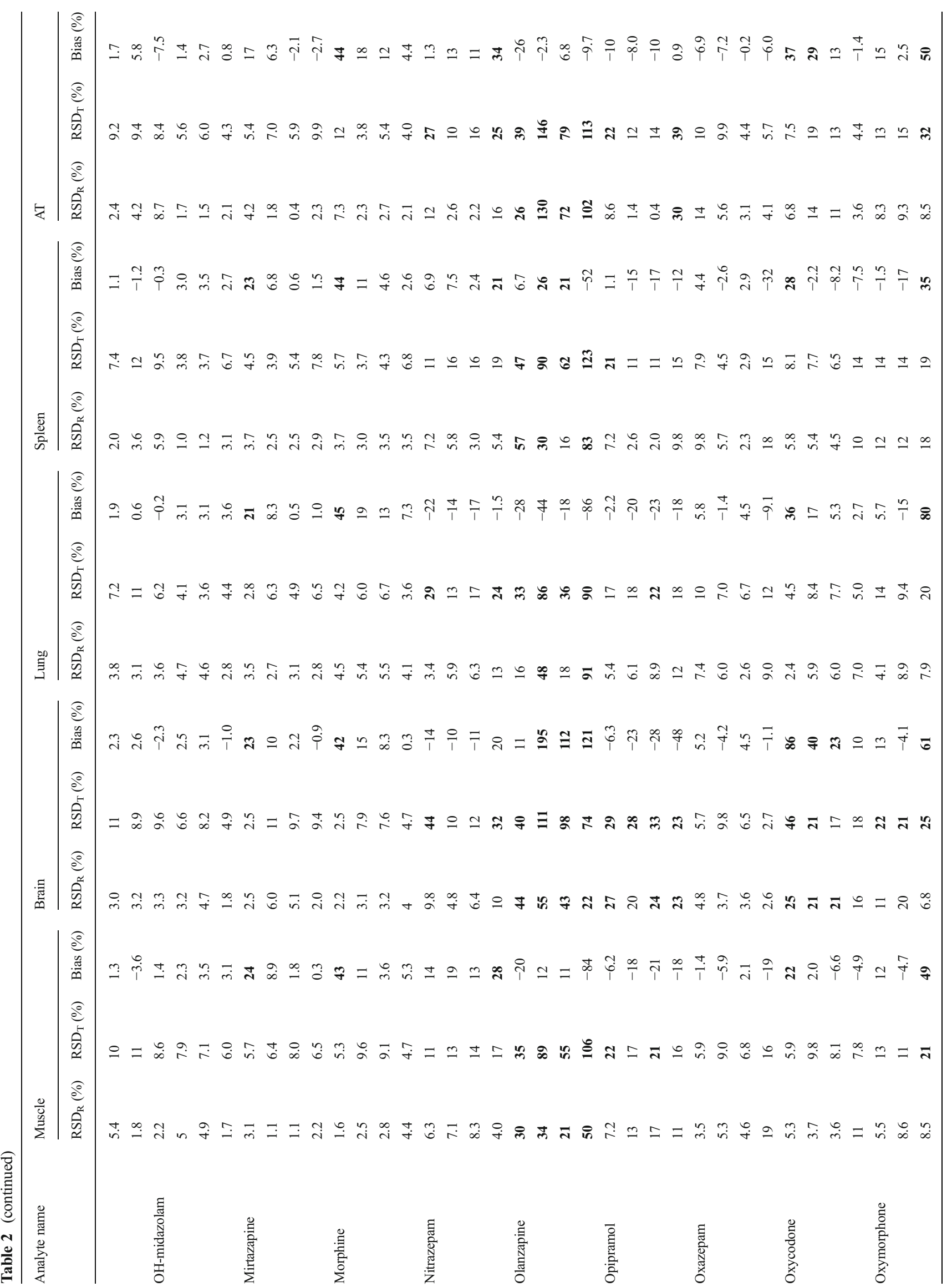




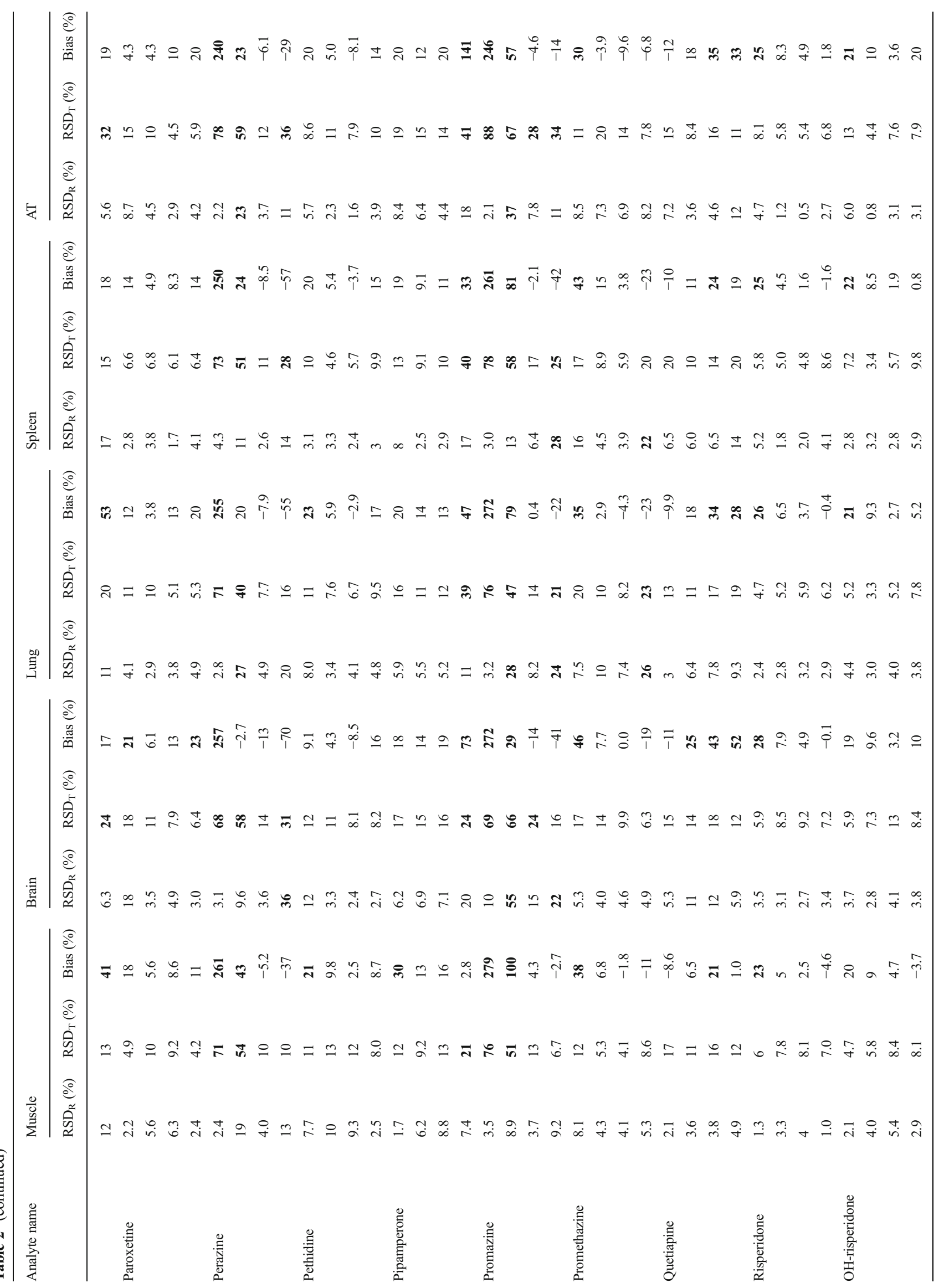




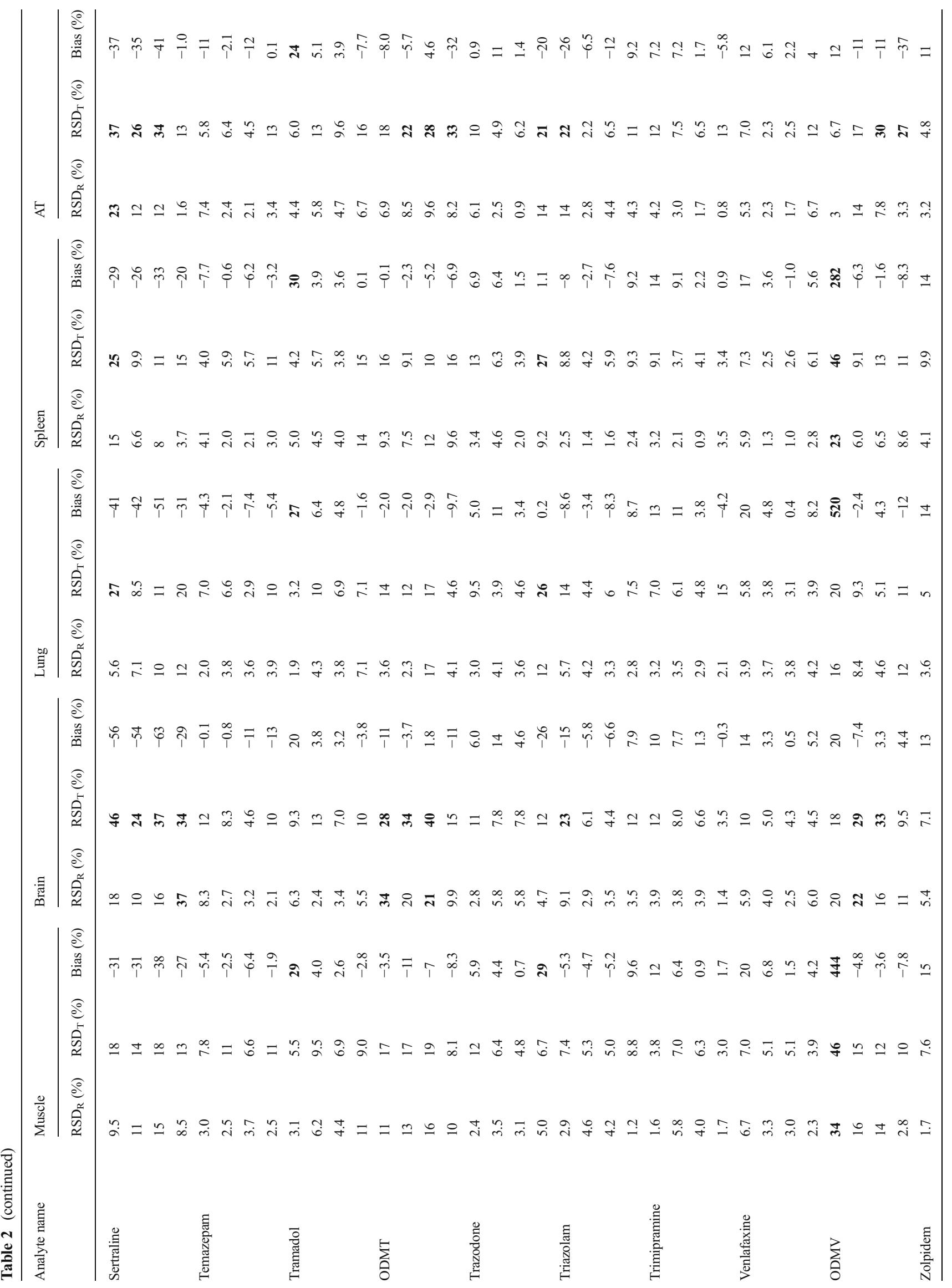


$20 \%$ for all QC samples, with exception of brain where only $75 \%$ of the analytes passed the criteria. As not always the same analytes failed, only 49 analytes (59\%) passed the criteria of the intra-day experiment in all ten matrices. Analytes failing in more than two matrices were bupropion, chlorprothixene, norclomipramine, flupentixol, flurazepam, $\mathrm{HC}$, levomepromazine, mCPP, EDDP, olanzapine, opipramol, perazine, pipamperone, promazine, promethazine, sertraline, and ODMV. All those analytes except mCPP and pipamperone did not have an IS with similar structure or retention time. In case of $\mathrm{mCPP}$ and pipamperone, the RTs of the IS mCPP-d8 and pipamperone-d10 shifted due to the rather high deuteration grade, which might have been responsible for the insufficient compensation. PMR for substances with low precision in a certain matrix will have to be interpreted with care-if reasonable interpretation will be possible at all — considering high analytical variations.

The inter-day precision seemed to be more matrix dependent than intra-day precision. In liver, brain, and AT, 60-64 \% of the analytes showed a precision below $20 \%$. In the other matrices, $76-83 \%$ of the analytes passed the criteria (Table 2). Also in this experiment, the intersection was smaller with only $43 \%$ of the analytes passing the criteria in all ten matrices. Additionally to the above-mentioned analytes, nortryptiline, OH-bupropion, clomipramine, norclozapine, nordoxepine, OH-flurazepam, lorazepam, MDA, nitrazepam, oxymorphone, quetiapine, ODMT, and trazodone failed the criteria in more than two matrices. Again, mainly analytes with no appropriate IS were affected.

In summary, all accuracy and precision parameters were fulfilled for 51 compounds in blood, 59 analytes in urine, 58 in CSF, 54 in muscle, 52 in kidney and spleen, 43 in liver, 42 in AT, and 41 in brain tissue. Twenty-eight analytes fulfilled all parameters in all ten matrices. Taking into consideration that so many analytes had been assessed in so many different matrices using a calibration in solvent instead of matrix and a calibration extension by ${ }^{13} \mathrm{C}$ isotopes, the overall performance in terms of accuracy and precision was perfectly acceptable.

\section{Limits}

The LOQs are listed in ESM Table S3. Although sample amount was a lot smaller compared with other quantitation methods used in forensic toxicology, LODs were in a comparable range $[14,34]$. As the detection of lower analyte concentrations was not of interest for the future application of the method, determination of LOD was resigned.

\section{Comparison of ${ }^{12} \mathrm{C}$ and ${ }^{13} \mathrm{C}$ calibrations}

In order to achieve a sufficient dynamic calibration range, two calibrations were combined: first, a conventional calibration with MRM $1\left({ }^{12} \mathrm{C}\right)$ for low to moderate concentrations, and 
second, an additional isotope calibration with a MRM $3\left({ }^{13} \mathrm{C}\right)$ for higher concentrations (Fig. 1). Applicability of this approach was tested by comparing accuracy of QC high determined using both calibrations. As exemplified for four matrices in Fig. 3, a difference in the calculated concentrations of QC high between the conventional and the isotope calibration greater than $20 \%$ was observed for five to seven analytes in each matrix. From the mentioned exceptions, levomepromazine, perazine, and promazine were above $20 \%$ in all and clomipramine, flupentixol, olanzapine, oxymorphone, and ODMV in more than two matrices. Although it seems as if the same analytes have similar errors in the different matrices (Fig. 3), no systematic findings were observed. For example, ODMV showed an error of $-30 \%$ in blood, $-20 \%$ in CSF, and + $25 \%$ in liver but was within $\pm 20 \%$ in all other matrices. Only levomepromazine, promazine, and oxymorphone showed a deviation in a similar range, so that changes in the calibration models might have improved the closeness between ${ }^{12} \mathrm{C}$ and ${ }^{13} \mathrm{C}$ calibrations. However, this might have worsened the bias for QC low or ${ }^{13} \mathrm{C}$. Generally, these analytes also showed problems in the other validation experiments, which may explain the deviations. Especially for these analytes, all samples of one case

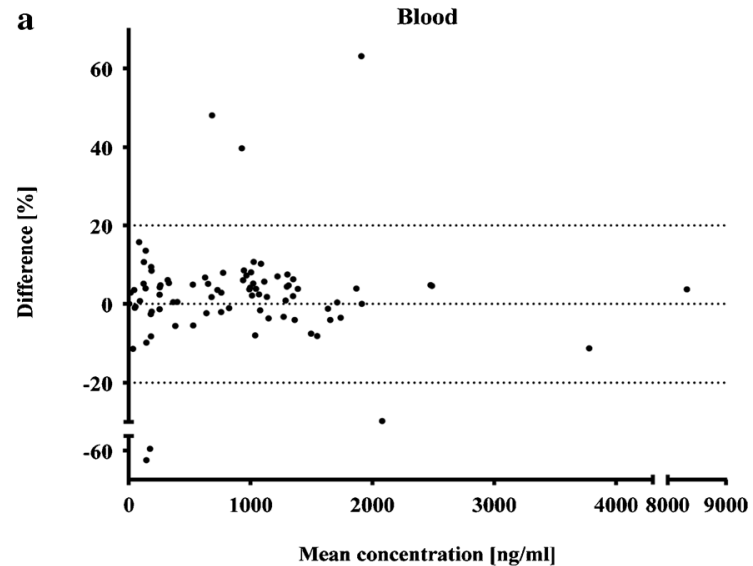

c

CSF

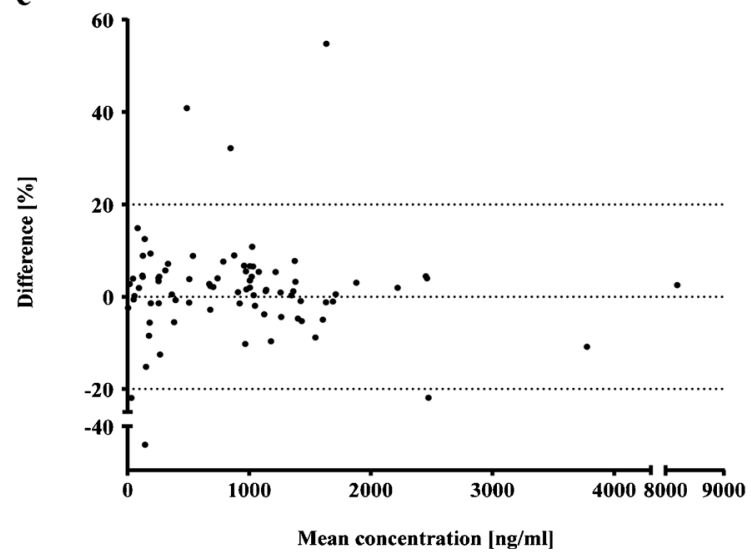

should be calculated either within ${ }^{12} \mathrm{C}$ or ${ }^{13} \mathrm{C}$ calibration. In general, combining ${ }^{12} \mathrm{C}$ and ${ }^{13} \mathrm{C}$ calibration successfully expanded the dynamic range without observing saturation effects.

\section{Conclusions}

An LC-MS/MS multi-analyte method for the simultaneous quantitation of 83 analytes in 11 postmortem matrices extracted from low milligram biopsy samples and small volumes of body fluids was developed with the aim to investigate concentration changes in postmortem samples taken at different time points. An extremely wide calibration range was achieved using a combination of a conventional $\left({ }^{12} \mathrm{C}\right)$ calibration for low to moderate concentrations and a ${ }^{13} \mathrm{C}$ isotope calibration of the same MRM for high concentrations. Calibration was successfully performed with external calibrators in solvent without matrix. The method was validated in terms of selectivity, crosstalk, ME, EE, accuracy, precision, and calibration model. Intra-day precision was the most important point in the validation process, considering the aim of concentration comparison between different time points in one matrix.
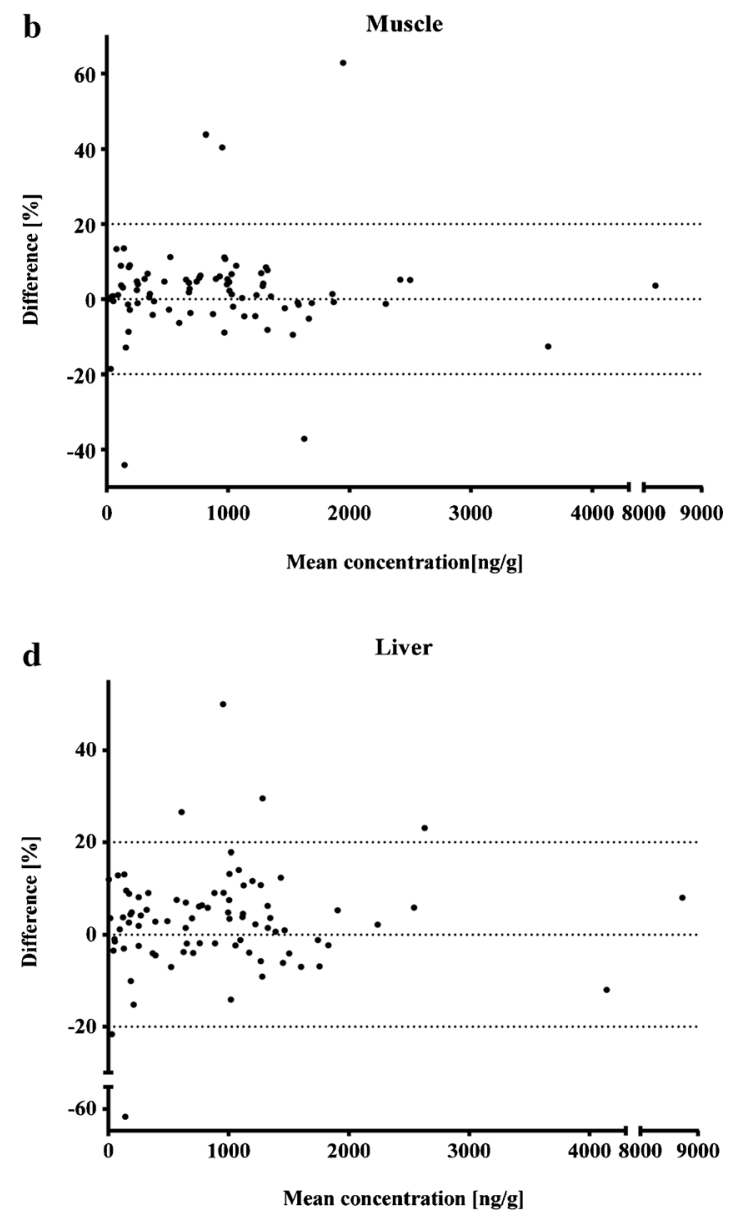

Fig. 3 Differences (\%) of the average QC high concentration calculated with ${ }^{12} \mathrm{C}$ calibration and ${ }^{13} \mathrm{C}$ calibration is depicted as Bland-Altman Plot of all analytes exemplarily in blood (a), muscle (b), CSF (c), and liver (d). Dotted lines represent deviation of $\pm 20 \%$ 
Depending on the matrix, $75-93 \%$ of the analytes fulfilled the criteria for a future study on time-dependent PMR, which is surprisingly good for such a universal method.

Acknowledgments The authors would like to thank Dr. Marc Chu and PD Dr. Frank T. Peters for their helpful discussions.

Conflict of interest The authors declare that they have no potential conflict of interest.

\section{References}

1. Pelissier-Alicot AL, Gaulier JM, Champsaur P, Marquet P (2003) Mechanisms underlying postmortem redistribution of drugs: a review. J Anal Toxicol 27(8):533-544

2. Jones GR, Pounder DJ (1987) Site dependence of drug concentrations in postmortem blood - a case study. J Anal Toxicol 11(5): $186-190$

3. Gerostamoulos D, Beyer J, Staikos V, Tayler P, Woodford N, Drummer OH (2012) The effect of the postmortem interval on the redistribution of drugs: a comparison of mortuary admission and autopsy blood specimens. Forensic Sci Med Pathol 8(4):373-379. doi:10.1007/s12024-012-9341-2

4. Skopp G (2004) Preanalytic aspects in postmortem toxicology. Forensic Sci Int 142(2-3):75-100

5. Noll BD, Coller JK, Somogyi AA, Morris RG, Russ GR, Hesselink DA, Van Gelder T, Sallustio BC (2013) Validation of an LC-MS/ MS method to measure tacrolimus in rat kidney and liver tissue and its application to human kidney biopsies. Ther Drug Monit 35(5): 617-623. doi:10.1097/FTD.0b013e31828e8162

6. Md Dom ZI, Noll BD, Coller JK, Somogyi AA, Russ GR, Hesselink DA, van Gelder T, Sallustio BC (2014) Validation of an LC-MS/MS method for the quantification of mycophenolic acid in human kidney transplant biopsies. J Chromatogr B Anal Technol Biomed Life Sci 945-946:171-177. doi:10.1016/j.jchromb.2013. 11.056

7. Maurer HH (2007) Analytical toxicology. Anal Bioanal Chem 388(7): 1311

8. Bonfigli AR, Manfrini S, Gregorio F, Testa R, Testa I, De Sio G, Coppa G (1999) Determination of plasma metformin by a new cation-exchange HPLC technique. Ther Drug Monit 21(3):330 334

9. Saar E, Gerostamoulos D, Drummer OH, Beyer J (2009) Comparison of extraction efficiencies and LC-MS-MS matrix effects using LLE and SPE methods for 19 antipsychotics in human blood. Anal Bioanal Chem 393(2):727-734. doi:10.1007/s00216008-2498-6

10. Maurer HH (2005) Multi-analyte procedures for screening for and quantification of drugs in blood, plasma, or serum by liquid chromatography-single stage or tandem mass spectrometry (LCMS or LC-MS/MS) relevant to clinical and forensic toxicology. Clin Biochem 38(4):310-318

11. Bergeron $M$, Bergeron $A$, van Amsterdam P, Furtado M, Garofolo F (2013) Use of polarity switching for the simultaneous bioanalysis of analytes with three orders of magnitude difference in concentration by LC-MS/MS. Bioanalysis 5(15):1911-1918. doi:10.4155/bio.13. 142

12. Curtis MA, Matassa LC, Demers R, Fegan K (2001) Expanding the linear dynamic range in quantitative high performance liquid chromatography/tandem mass spectrometry by the use of multiple product ions. Rapid Commun Mass Spectrom: RCM 15(12):963968. doi:10.1002/rcm.327
13. Hoefs J (2013) Stable isotope geochemistry. Springer Science \& Business Media

14. Steuer AE, Poetzsch M, Koenig M, Tingelhoff E, Staeheli SN, Roemmelt AT, Kraemer T (2015) Comparison of conventional liquid chromatography-tandem mass spectrometry versus microflow liquid chromatography-tandem mass spectrometry within the framework of full method validation for simultaneous quantification of 40 antidepressants and neuroleptics in whole blood. J Chromatogr A 1381:87-100. doi:10.1016/j.chroma.2014.12.084

15. Matuszewski BK, Constanzer ML, Chavez-Eng CM (2003) Strategies for the assessment of matrix effect in quantitative bioanalytical methods based on HPLC-MS/MS. Anal Chem 75(13):3019-3030

16. Peters FT, Hartung M, Herbold M, Schmitt G, Daldrup T, Musshoff F (2009) Anhang B zur Richtlinie der GTFCh zur Qualitätssicherung bei forensisch-toxikologischen Untersuchungen. Anforderungen an die Validierung von Analysenmethoden. Toxichem Krimtech 76:185

17. Eberhard J, Jepsen S, Albers HK, Acil Y (2000) Quantitation of arachidonic acid metabolites in small tissue biopsies by reversedphase high-performance liquid chromatography. Anal Biochem 280(2):258-263. doi:10.1006/abio.2000.4540

18. Szekely-Klepser G, Wade K, Woolson D, Brown R, Fountain S, Kindt E (2005) A validated LC/MS/MS method for the quantification of pyrrole-2,3,5-tricarboxylic acid (PTCA), a eumelanin specific biomarker, in human skin punch biopsies. J Chromatogr B Anal Technol Biomed Life Sci 826(1-2):31-40. doi:10.1016/j. jchromb.2005.08.002

19. Frost J, Lokken TN, Brede WR, Hegstad S, Nordrum IS, Slordal L (2015) A validated method for simultaneous determination of codeine, codeine-6-glucuronide, norcodeine, morphine, morphine-3glucuronide and morphine-6-glucuronide in post-mortem blood, vitreous fluid, muscle, fat and brain tissue by LC-MS. J Anal Toxicol 39(3):203-212. doi:10.1093/jat/bku145

20. Luckenbill K, Thompson J, Middleton O, Kloss J, Apple F (2008) Fentanyl postmortem redistribution: preliminary findings regarding the relationship among femoral blood and liver and heart tissue concentrations. J Anal Toxicol 32(8):639-643

21. Roman M, Kronstrand R, Lindstedt D, Josefsson M (2008) Quantitation of seven low-dosage antipsychotic drugs in human postmortem blood using LC-MS-MS. J Anal Toxicol 32(2):147155

22. Bonfiglio R, King RC, Olah TV, Merkle K (1999) The effects of sample preparation methods on the variability of the electrospray ionization response for model drug compounds. Rapid Commun Mass Spectrom: RCM 13(12):1175-1185. doi:10.1002/(SICI) 1097-0231(19990630)13:12<1175::AID-RCM639>3.0.CO;2-0

23. Bynum ND, Poklis JL, Gaffney-Kraft M, Garside D, Ropero-Miller JD (2005) Postmortem distribution of tramadol, amitriptyline, and their metabolites in a suicidal overdose. J Anal Toxicol 29(5):401406

24. Hansen KC, Schmitt-Ulms G, Chalkley RJ, Hirsch J, Baldwin MA, Burlingame AL (2003) Mass spectrometric analysis of protein mixtures at low levels using cleavable $13 \mathrm{C}$-isotope-coded affinity tag and multidimensional chromatography. Mol Cell Proteome: MCP 2(5):299-314. doi:10.1074/mcp.M300021-MCP200

25. Haubl G, Berthiller F, Krska R, Schuhmacher R (2006) Suitability of a fully $13 \mathrm{C}$ isotope labeled internal standard for the determination of the mycotoxin deoxynivalenol by LC-MS/MS without clean up. Anal Bioanal Chem 384(3):692-696. doi:10.1007/s00216-0050218-Z

26. Meier-Augenstein W (1999) Applied gas chromatography coupled to isotope ratio mass spectrometry. J Chromatogr A 842(1-2):351371

27. Remane D, Meyer MR, Wissenbach DK, Maurer HH (2010) Ion suppression and enhancement effects of co-eluting analytes in 
multi-analyte approaches: systematic investigation using ultrahigh-performance liquid chromatography/mass spectrometry with atmospheric-pressure chemical ionization or electrospray ionization. Rapid Commun Mass Spectrom: RCM 24(21):3103-3108. doi: $10.1002 / \mathrm{rcm} .4736$

28. Peters FT, Drummer OH, Musshoff F (2007) Validation of new methods. Forensic Sci Int 165(2-3):216-224

29. Annesley TM (2003) Ion suppression in mass spectrometry. Clin Chem 49(7):1041-1044

30. Dams R, Huestis MA, Lambert WE, Murphy CM (2003) Matrix effect in bio-analysis of illicit drugs with LC-MS/MS: influence of ionization type, sample preparation, and biofluid. J Am Soc Mass Spectrom 14(11):1290-1294

31. Olesen OV, Linnet K (1998) Determination of olanzapine in serum by high-performance liquid chromatography using ultraviolet detection considering the easy oxidability of the compound and the presence of other psychotropic drugs. J Chromatogr B Biomed Sci Appl 714(2):309-315

32. Saar E, Gerostamoulos D, Drummer OH, Beyer J (2012) Identification of 2-hydroxymethyl-olanzapine as a novel degradation product of olanzapine. Forensic Sci Int 220(1-3):74-79. doi: 10.1016/j.forsciint.2012.01.029

33. Hayen H, Karst U (2003) Analysis of phenothiazine and its derivatives using LC/electrochemistry/MS and LC/electrochemistry/ fluorescence. Anal Chem 75(18):4833-4840

34. Steuer AE, Forss AM, Dally AM, Kraemer T (2014) Method development and validation for simultaneous quantification of 15 drugs of abuse and prescription drugs and 7 of their metabolites in whole blood relevant in the context of driving under the influence of drugs - usefulness of multi-analyte calibration. Forensic Sci Int 244:92-101. doi:10.1016/j. forsciint.2014.08.022 